Issued by Sandia National Laboratories, operated for the United States Department of Energy by Sandia Corporation.

NOTICE: This report was prepared as an account of work sponsored by an agency of the United States Government. Neither the United States Government, nor any agency thereof, nor any of their employees, nor any of their contractors, subcontractors, or their employees, make any warranty, express or implied, or assume any legal liability or responsibility for the accuracy, completeness, or usefulness of any information, apparatus, product, or process disclosed, or represent that its use would not infringe privately owned rights. Reference herein to any specific commercial product, process, or service by trade name, trademark, manufacturer, or otherwise, does not necessarily constitute or imply its endorsement, recommendation, or favoring by the United States Government, any agency thereof, or any of their contractors or subcontractors. The views and opinions expressed herein do not necessarily state or reflect those of the United States Government, any agency thereof, or any of their contractors.

Printed in the United States of America. This report has been reproduced directly from the best available copy.

Available to DOE and DOE contractors from

Office of Scientific and Technical Information

P.O. Box 62

Oak Ridge, TN 37831

Prices available from (703) 605-6000

Web site: http://www.ntis.gov/ordering.htm

Available to the public from

National Technical Information Service

U.S. Department of Commerce

5285 Port Royal Rd

Springfield, VA 22161

NTIS price codes

Printed copy: A03

Microfiche copy: A01

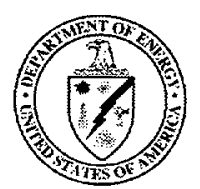




\section{DISCLAIMER}

Portions of this document may be illegible in electronic image products. Images are produced from the best available original document. 
SAND99-1739

Unlimited Release

Printed July 1999

\title{
Analysis of Subsidence Data for the Bryan Mound Site, Texas
}

\author{
Stephen J. Bauer \\ Underground Storage Technology \\ Sandia National Laboratories \\ P.O. Box 5800 \\ Albuquerque, NM 87185-0706
}

\begin{abstract}
The elevation change data measured at the Bryan Mound Strategic Petroleum Reserve (SPR) site over the last $16+$ years has been studied and a model utilized to project elevation changes into the future. The subsidence rate at Bryan Mound is low in comparison with other Strategic Petroleum Reserve sites and has decreased with time due to the maintenance of higher operating pressures and the normal decrease in creep closure rate of caverns with time. However, the subsidence at the site is projected to continue. A model was developed to project subsidence values 20 years into the future; no subsidence related issues are apparent from these projections.
\end{abstract}




\section{Table of Contents}

Page

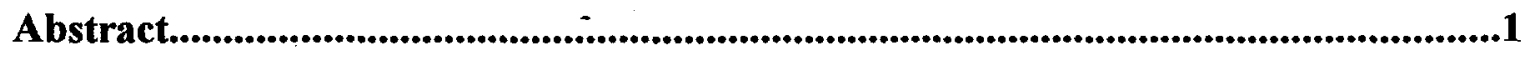

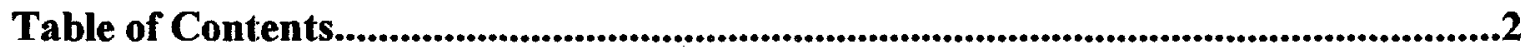

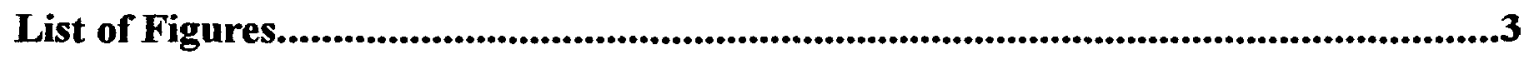

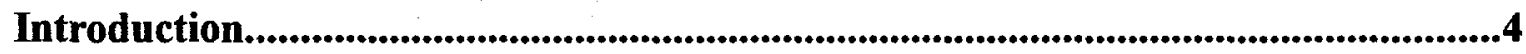

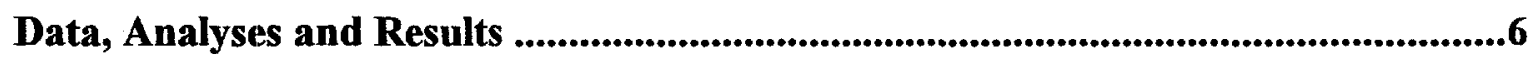

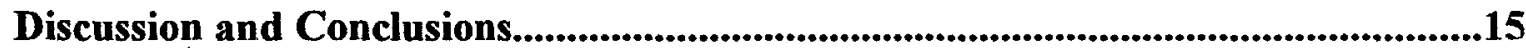

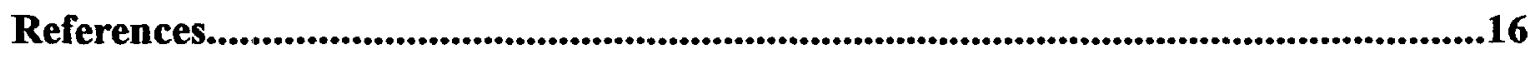

Appendix 1: Measured and Projected Elevations at Bryan Mound..........................17

Appendix 2: Calculated Historical Subsidence Rates at Bryan Mound....................19

Appendix 3: Fitting Parameters for Long Term Subsidence Prediction.....................25

Distribution....................................................................................................27 


\section{List of Figures}

Figure $\quad$ Page

1. Top of cap rock contour map at Bryan Mound..........................................5

2. Bryan Mound site measured elevations (feet), January, 1999.............................6

3. Bryan Mound site measured elevations (feet), February, 1991........................7

4. Bryan Mound site measured elevations (feet), December, 1982.......................7

5. Bryan Mound subsidence rate, 12/82-12/88 (ft/yr)....................................9

6. Bryan Mound subsidence rate, 12/88-4/94 (ft/yr) ............................................9

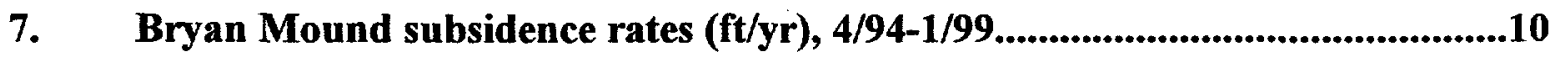

8. Bryan Mound subsidence rates (ft/yr), 2/97-1/99.........................................10

9. Examples of the exponential decay fit for stations "10C" and "BM 5"..........12

10. Bryan Mound site projected elevations (feet) for the year 2004......................13

11. Bryan Mound site projected elevations (feet) for the year 2009......................14

12. Bryan Mound site projected elevations (feet) for the year 2019......................14 


\section{Introduction}

The subsidence monument elevations at the Bryan Mound Strategic Petroleum Reserve (SPR) site have been surveyed nine times beginning in December, 1982. The earlier survey data have been most recently reported on by Osnes (1995). This report provides an update which includes additional measurements completed in the past few years. The changes in elevation, the rates of subsidence, as well as projections of future elevation changes are presented. Elevation data were most recently collected at the Bryan Mound site in January 1999. Data of this type is important and has been used by the cavern engineer and other site personnel in assuring certain aspects of site integrity. A layout of the site with caverns and the DOE property boundary is given in Figure 1.

At Bryan Mound and SPR sites in general, elevation changes are measured because they document surface subsidence resulting from creep closure of caverns. General subsidence on the scale of the site or portions thereof is seen in the survey data taken. This type of subsidence will capture gross effects of creep closure of underground openings in response to the state of stress. Survey measurements are taken at the site are every other year. However, the detailed long term subsidence provided by the surveys is important, especially because it permits the long term extrapolation of elevation changes into the future.

Elevation data represents the raw data. The most recent data set included 65 data points. The number of data points varies from year to year because it is a function of ability to find monuments, destruction of monuments, damage to monuments, etc. This year 24 monuments were repositioned, therefore data for the past two years has been lost at these positions, and another three monuments were destroyed; as a result a total of about a third of the possible data at Bryan Mound was lost for this measurement period. The measurements have been made at various time intervals; the current time interval was about two years.

In practice, measurements of subsidence are difficult at best. At Bryan Mound the reference is an off-site benchmark. This has the potential to introduce some small error in traversing the distance to the site. The leveling surveys are performed to Second-Order First-Class accuracy, with allowable vertical closure not to exceed approximately $0.025 \mathrm{ft} / \mathrm{mile}^{0.5}$ (Osnes, 1995). 


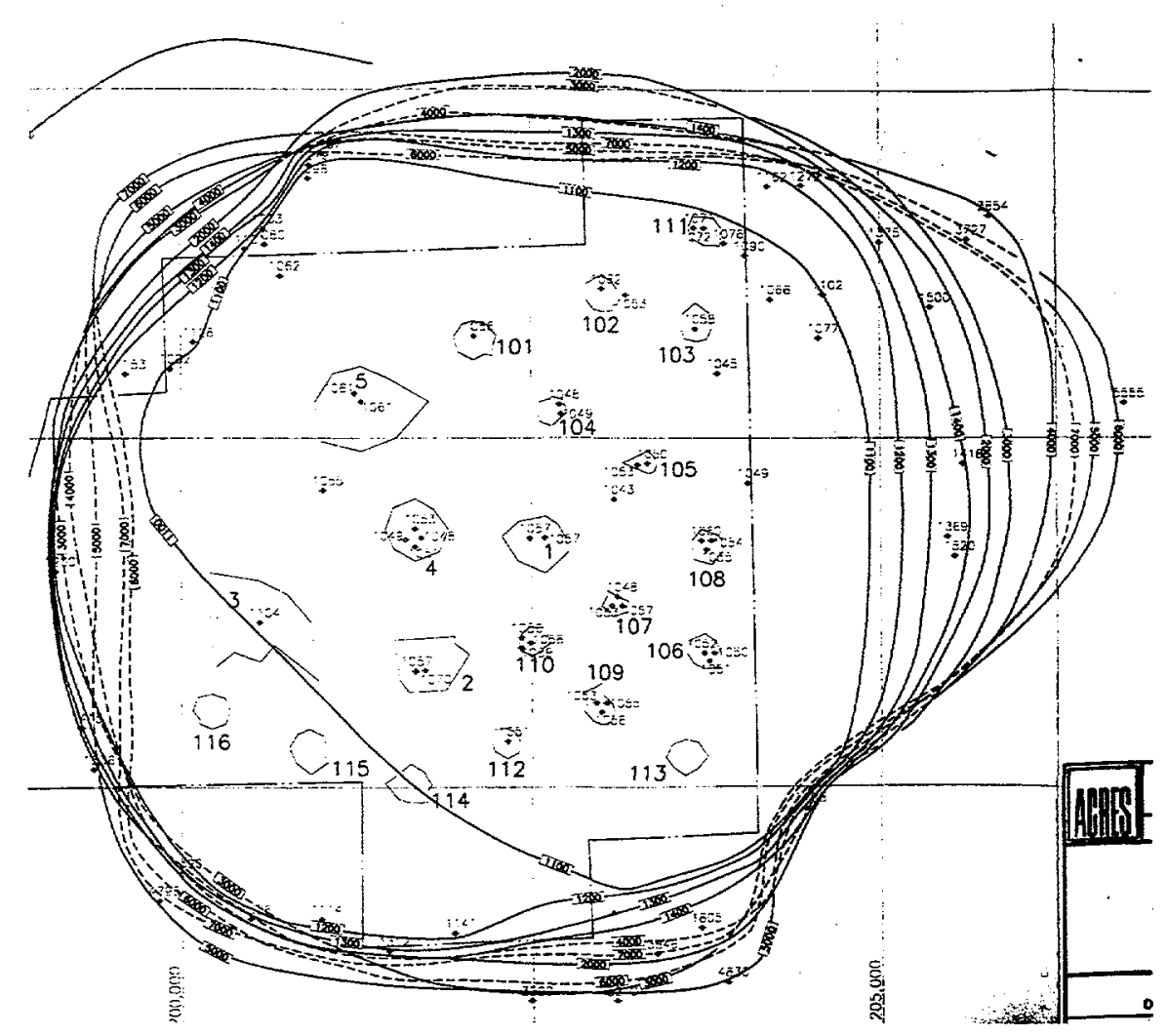

Figure 1. Top of salt rock contour map at Bryan Mound. From Neal, et al., 1994, "Strategic Petroleum Reserve (SPR) Additional Geologic Site Characterization Studies Bryan Mound Salt Dome, Texas," SAND94-2331. Also shown are cavern location and the DOE property boundary.

The Bryan Mound site is generally low in elevation and is susceptible to flooding. On the southeast are Mud Lake, the Intracoastal Waterway, and the Gulf of Mexico, on the west is the New Brazos River, and on the northwest is Blue Lake. The caprock at Bryan Mound is approximately 300 feet thick with some minor faulting. The effect of this caprock's thickness and structure is an unknown. The extensive area at or below 10 feet of elevation and the proximity to water represent important factors in studying the subsidence at this site. The historic elevation of the Bryan Mound site and evidence of the progressive subsidence at the site are shown in Figures 2, 3, and 4. Significantly, during the past 16 years the extent of the site area with an elevation less than 10 feet has nearly doubled (from $25 \%$ to near $50 \%$ ). 


\section{Data, Analyses, and Results}

Elevation data is reported in Appendix 1 and portrayed in Figures 2-4. Some stations are located on well heads, thus the elevations reported are for well head elevations, not the ground level. This location of stations on well heads is common for SPR sites, because of the convenience. The January, 1999 elevation data are presented in Figure 2. Elevations recently reported (Figure 2) are generally consistent with the projected continual slow subsidence of the site. Twenty-four survey points were recently repositioned (vertically) during site maintenance activities and are shown in red. As a result of the maintenance, these data cannot be used in the current analysis. Beginning with the next survey these points can be used in assessing the elevation change at these locations. Later in the report, analyses are presented which obviate the immediate need for these data. However, routine loss of this many data points from survey to survey would eventually compromise the ability to provide meaningful analyses and thus would limit the ability to make good projections of elevations into the future.

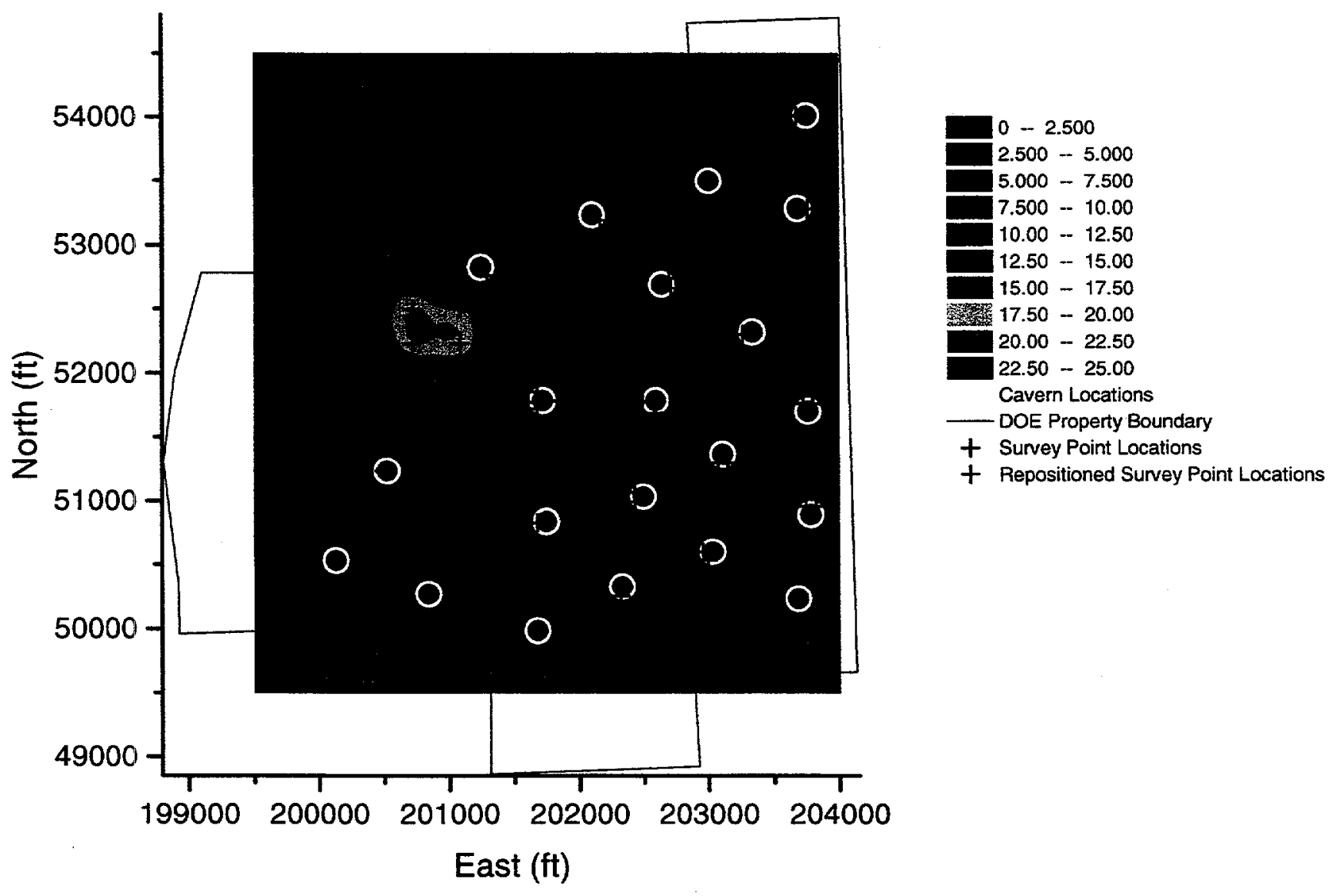

Figure 2. Bryan Mound site measured elevations (feet), January,1999. The contour interval is 2.5 feet. 


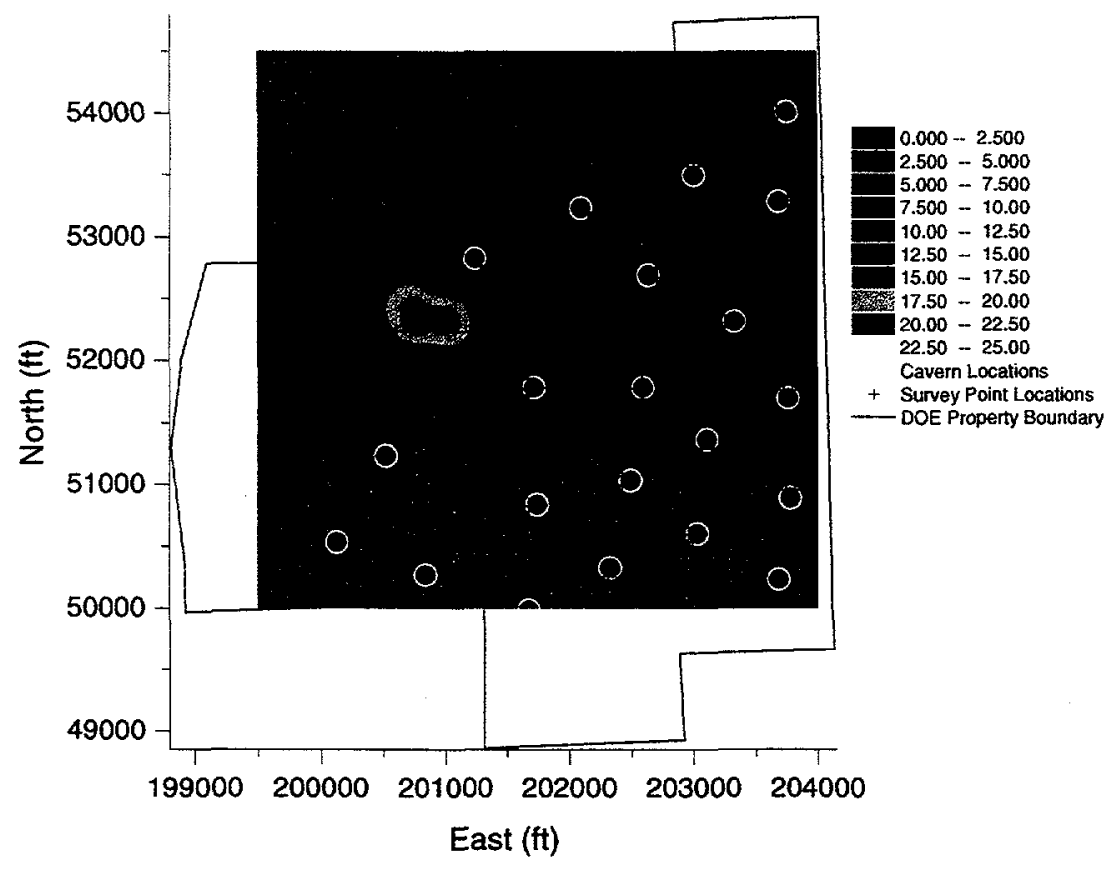

Figure 3. Bryan Mound site measured elevations (feet), February, 1991. The contour interval is 2.5 feet.

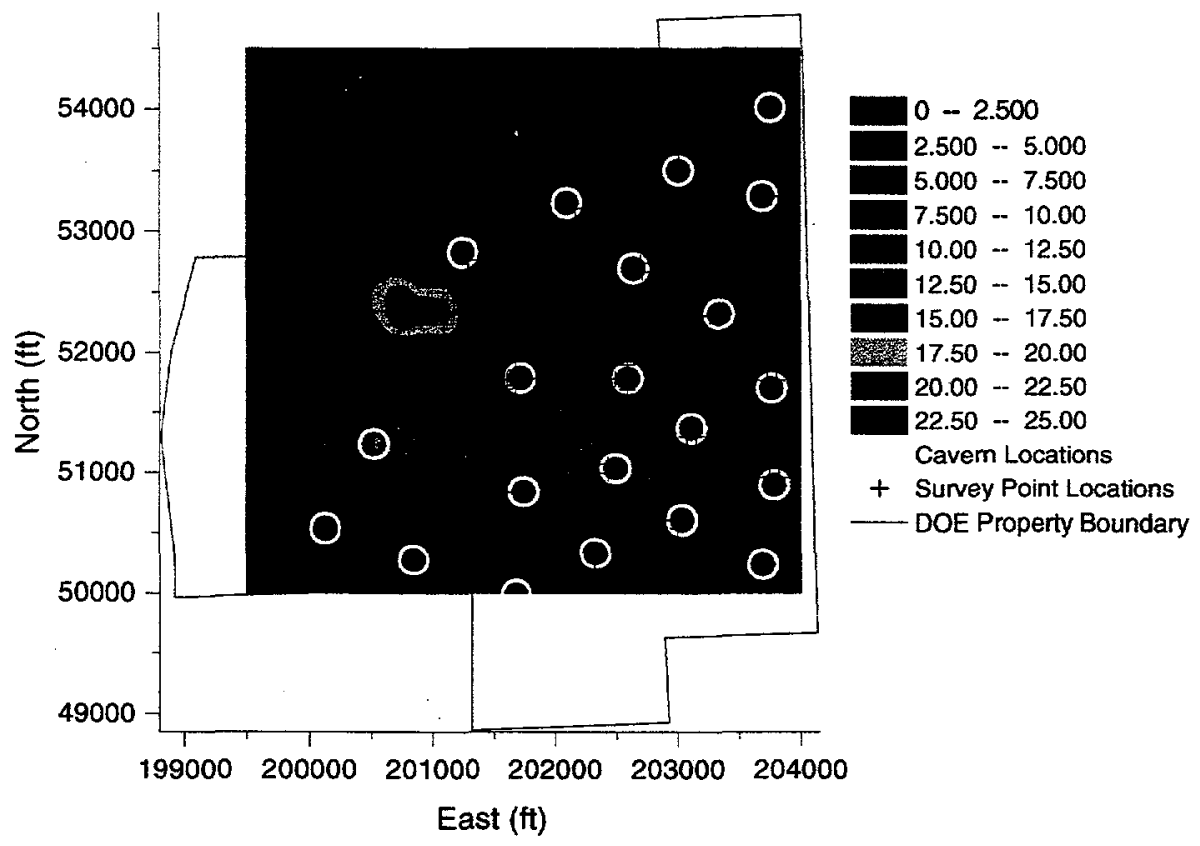

Figure 4. Bryan Mound site measured elevations (feet), December, 1982. The contour interval is 2.5 feet. 
The rate of subsidence was studied to determine the uniformity of subsidence rates across the site. Local areas of greater subsidence rates may be signals of abnormal creep closure behavior of the underlying SPR caverns. The rate of subsidence is calculated by dividing the amount of elevation change in a time period by the time span of the period in years. This calculation was made for each time period between measurements and is presented in Appendix 2. The calculation was also made for longer time periods and results are presented in Figures 5, 6, and 7. The long time periods should incorporate the variation from one measurement to the next that sometimes marks variability in the subsidence rate (Appendix 2).

The rate of subsidence has decreased during the measurement period. For the first six years of measurement (1982-1988) the site subsidence rate ranged from 0.08 to 0.12 feet per year (Figure 5); for the next five years the rate decreased to 0.02 to 0.08 inches per year (Figure 6). For the most recent five year time period, the rate has decreased further and is now closer to a 0.01 to 0.05 feet per year value (Figure 7 ).

The decrease observed is probably due to at least two reasons, the operational procedure adopted of maintaining the caverns at relatively high operating pressure and the corresponding decrease in creep closure rate of the caverns. Transient creep effects also diminishes the closure rate with time (Ehgartner, 1992).

Some monuments at each site are on well heads, some are "deeply rooted" and some are attached to surface elements (for example light poles, buildings, etc.). All of these types of monuments are valuable to the elevation monitoring array at a site. Those monuments attached to surface elements may be effected by near surface phenomena, for example water level changes, although this effect has never been proven. Monuments that sit on well heads are tied by the casing to the top of the cavern. These monuments likely record the effect of deeply seated displacements.

In each of the time periods portrayed, the subsidence rate is slightly greater in the southern (Figure 6) and southwestern portions, (Figures 7 and 8) of the site. This appears to be a consistent trend. For the most recent two year measurement period (2/97-1/99), that greater rate area appears to centered in an area that extends from Cavern 3 (plugged and abandoned) east to Cavern 2, and northeast from Cavern 3 about 1000'. The most recent value of the subsidence rate at Cavern 3 is a little more than 0.125 feet per year. While this remains a relatively small value in comparison to other SPR sites, it does represent a statistically significant change from the previous time period, and is large compared to historical values at Bryan Mound. These measurements may be the result of error, periodic measurement variations (which have occurred in the past), or they could be "real". However, this observation may be signaling an area at the site that should be watched more closely until the next survey (about 24 months). Both Caverns 3 and 2 are shallow (top near $-1500 \mathrm{ft}$ ) and somewhat pancake shaped. 


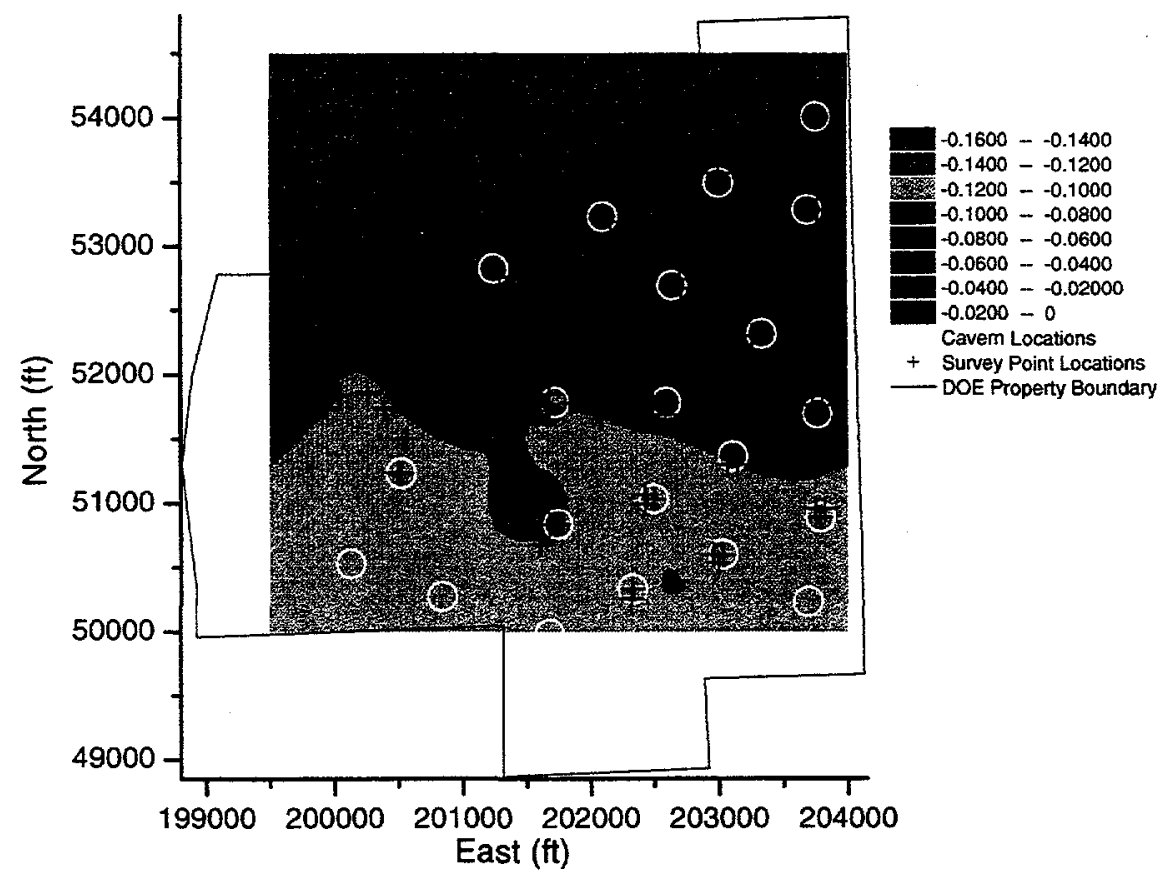

Figure 5. Bryan Mound subsidence rate, 12/82-12/88 (ft/yr). The contour interval is $0.02 \mathrm{ft} / \mathrm{yr}$.

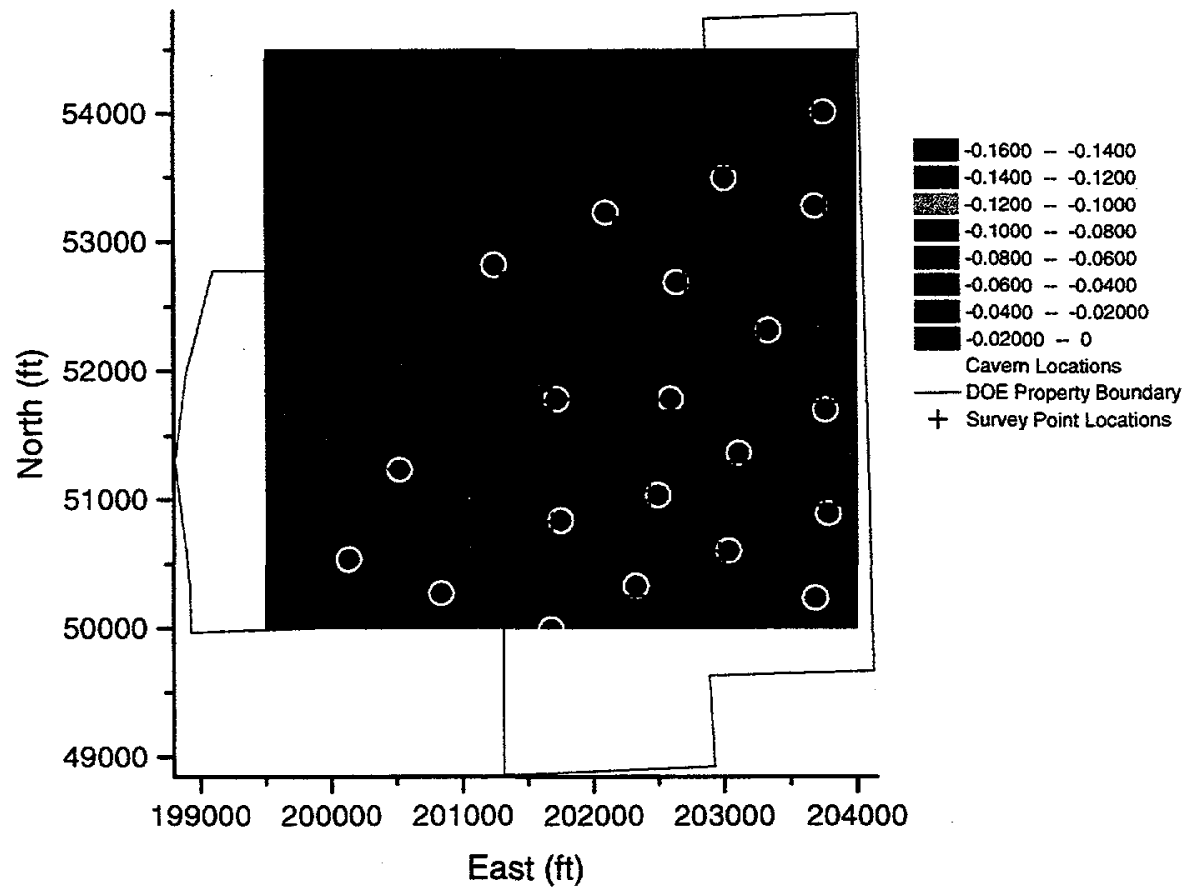

Figure 6. Bryan Mound subsidence rate, $12 / 88-4 / 94$ (ft/yr). The contour interval is $0.02 \mathrm{ft} / \mathrm{yr}$. 


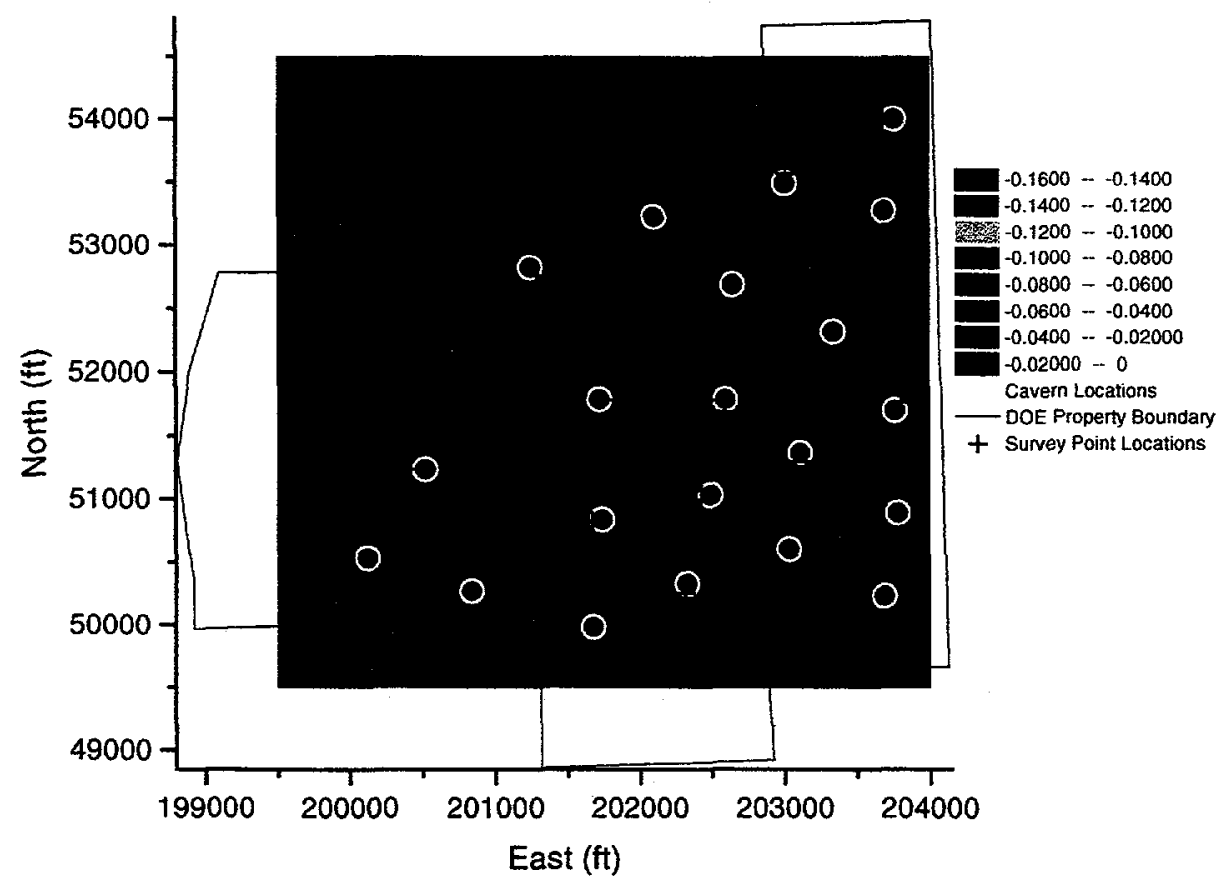

Figure 7. Bryan Mound Subsidence Rates (ft/yr), 4/94-1/99. The contour interval is $0.02 \mathrm{ft} / \mathrm{yr}$.

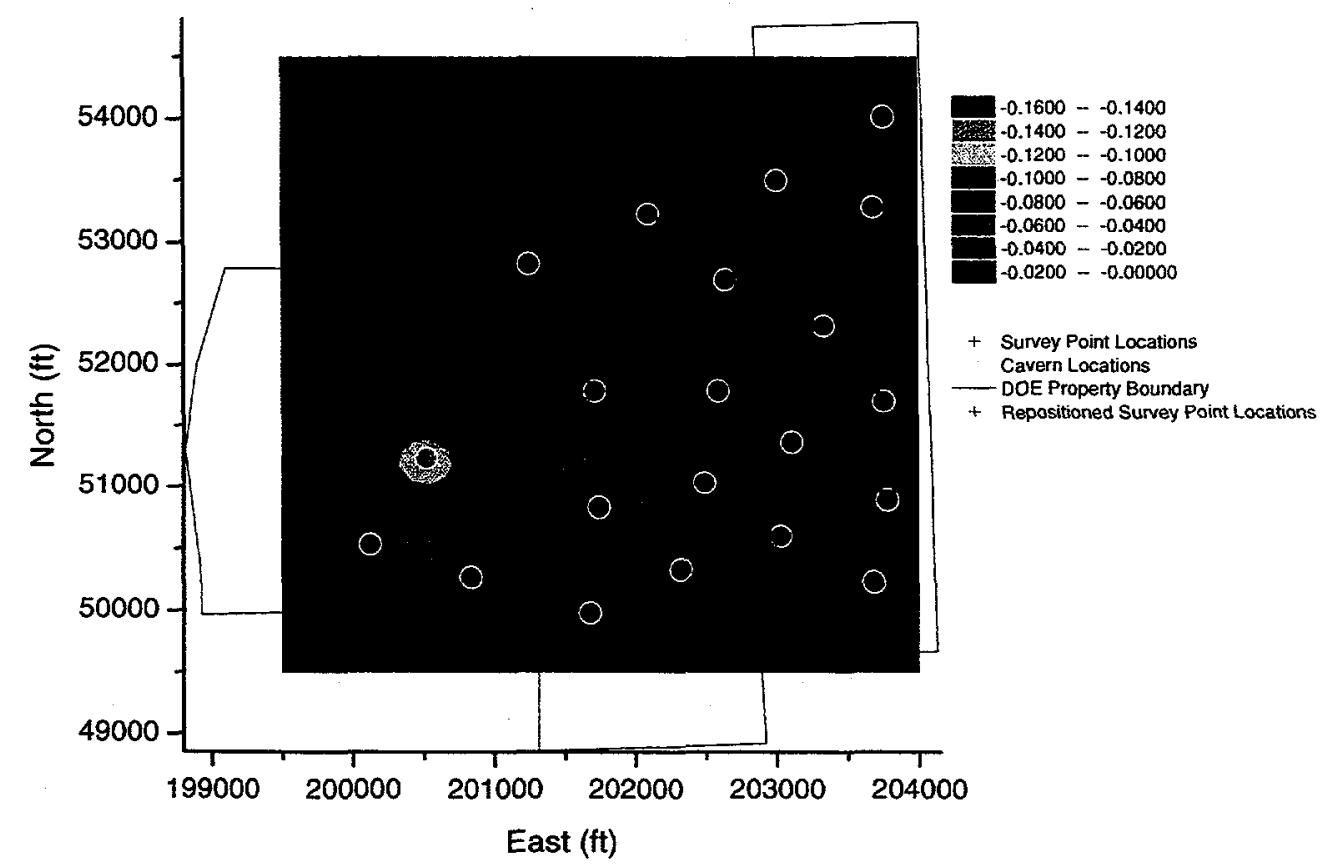

Figure 8. Bryan Mound Subsidence Rates (ft/yr), 2/97-1/99. The contour interval is $0.02 \mathrm{ft} / \mathrm{yr}$. 
It is important to develop a relationship to extrapolate changes in elevation into the future, in order to provide the DOE with important information for planning future work at the site. Such an extrapolation is possible by making use of the past history of the elevation measurements. Elevation data collected at known times at each station were fit to a first order exponential decay of the form:

$$
Y=Y_{0}+A_{1} e^{-\left(x-x_{0}\right) / t_{1}}
$$

where $Y$ is the calculated elevation at the time of interest $(x), Y_{0}$ is the elevation at $X_{0}($ time $=0)$, $A_{1}$ is a constant, and $t_{1}$ is a fitting parameter.

This relationship allows the data to be projected into the future with confidence. Examples of the data compared to the first order exponential decay relationship are given in Figure 10. Appendix 3 contains fitting parameters for equations fit to data for each measurement station allowing the reader to make projections to any time in the future. 

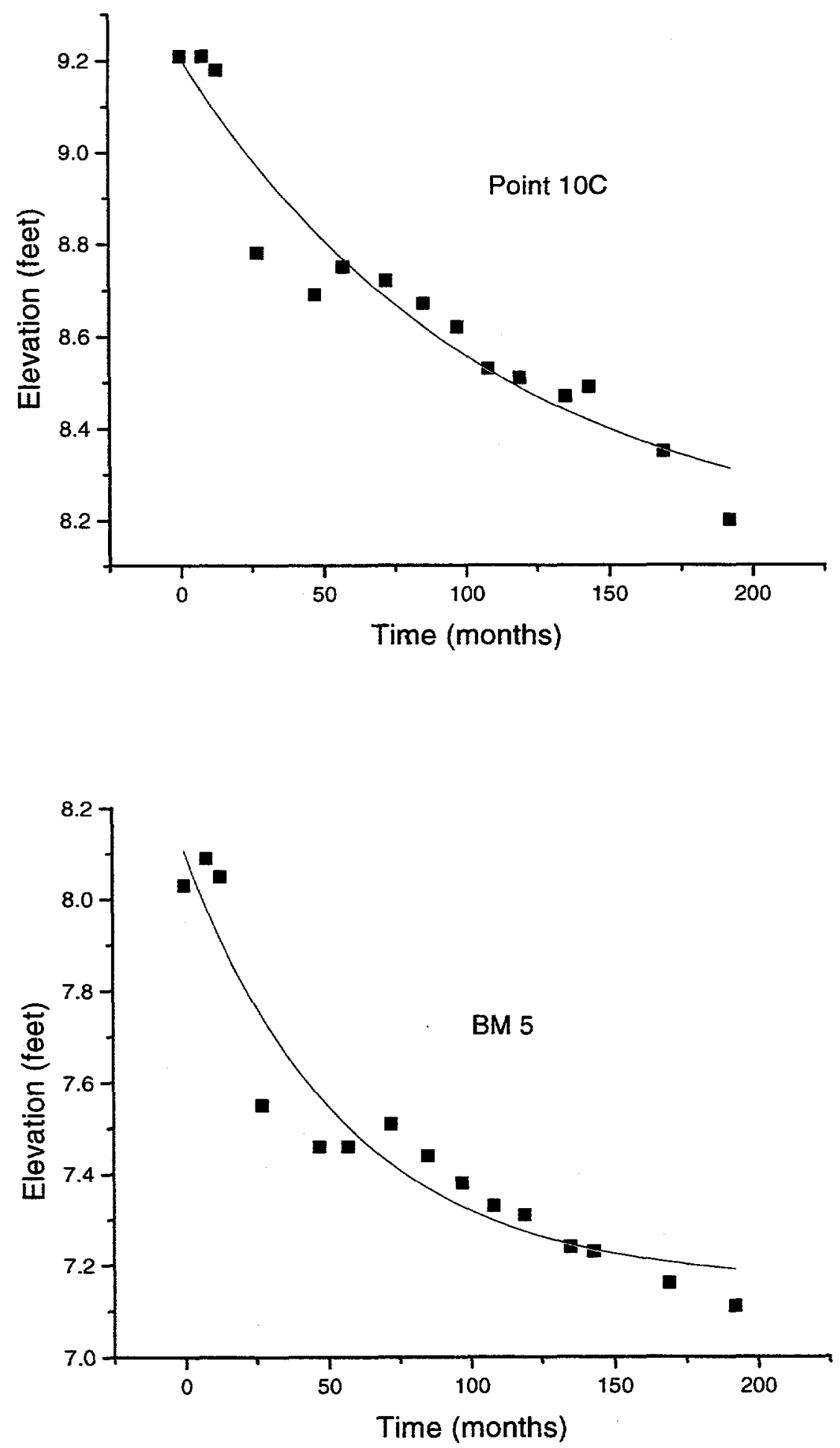

Figure 9. Examples of the exponential decay fit for stations "10C" and "BM 5". 
Projected elevations for the years 2004, 2009, and 2019 are presented in Figures 9, 10, and 11. Consistent with information already presented, it is predicted that the Bryan Mound site will continue to subside, but at an ever decreasing rate in accordance with the first order exponential decay.

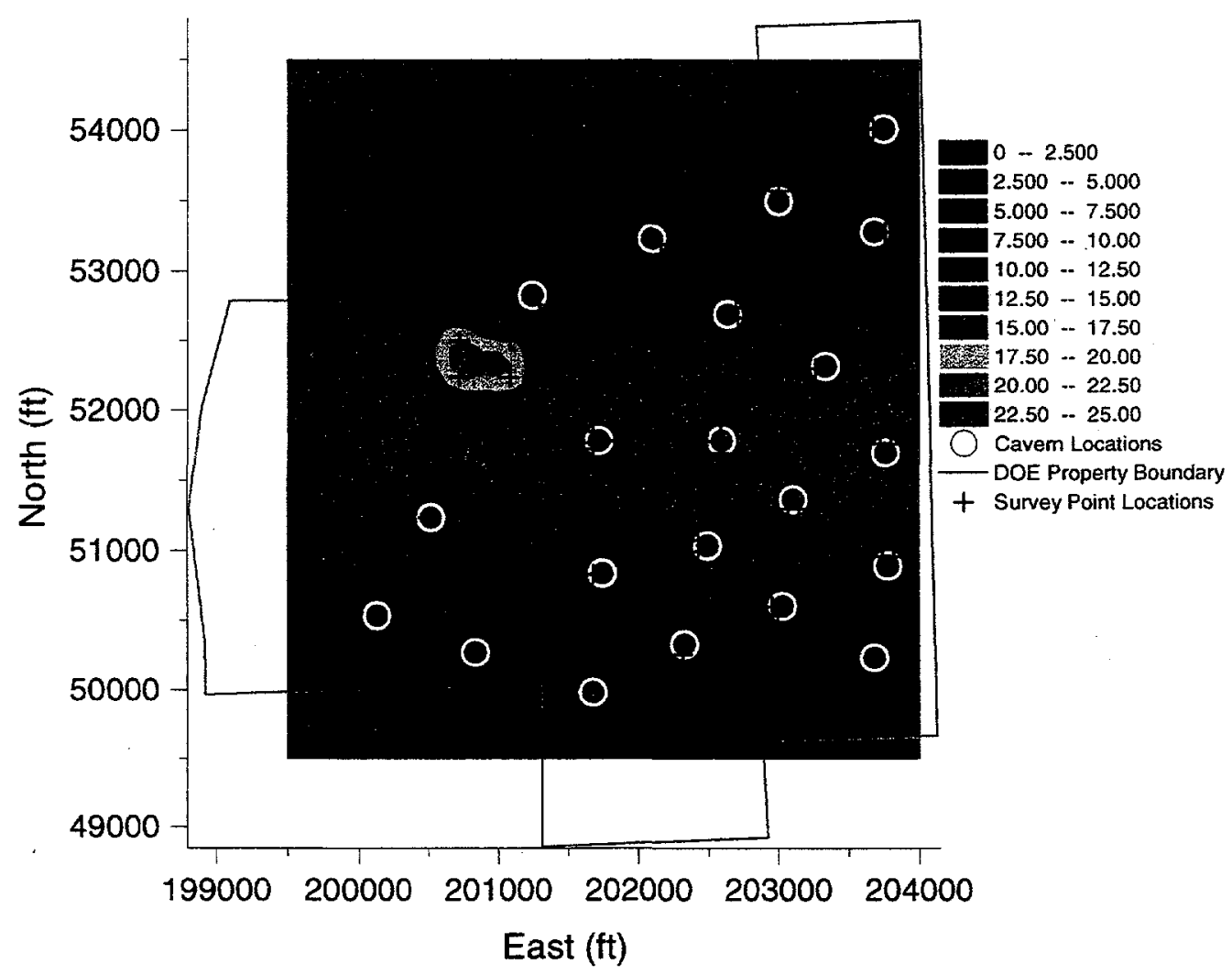

Figure 10. Bryan Mound site projected elevations (feet) for the year 2004. The contour interval is 2.5 feet. 


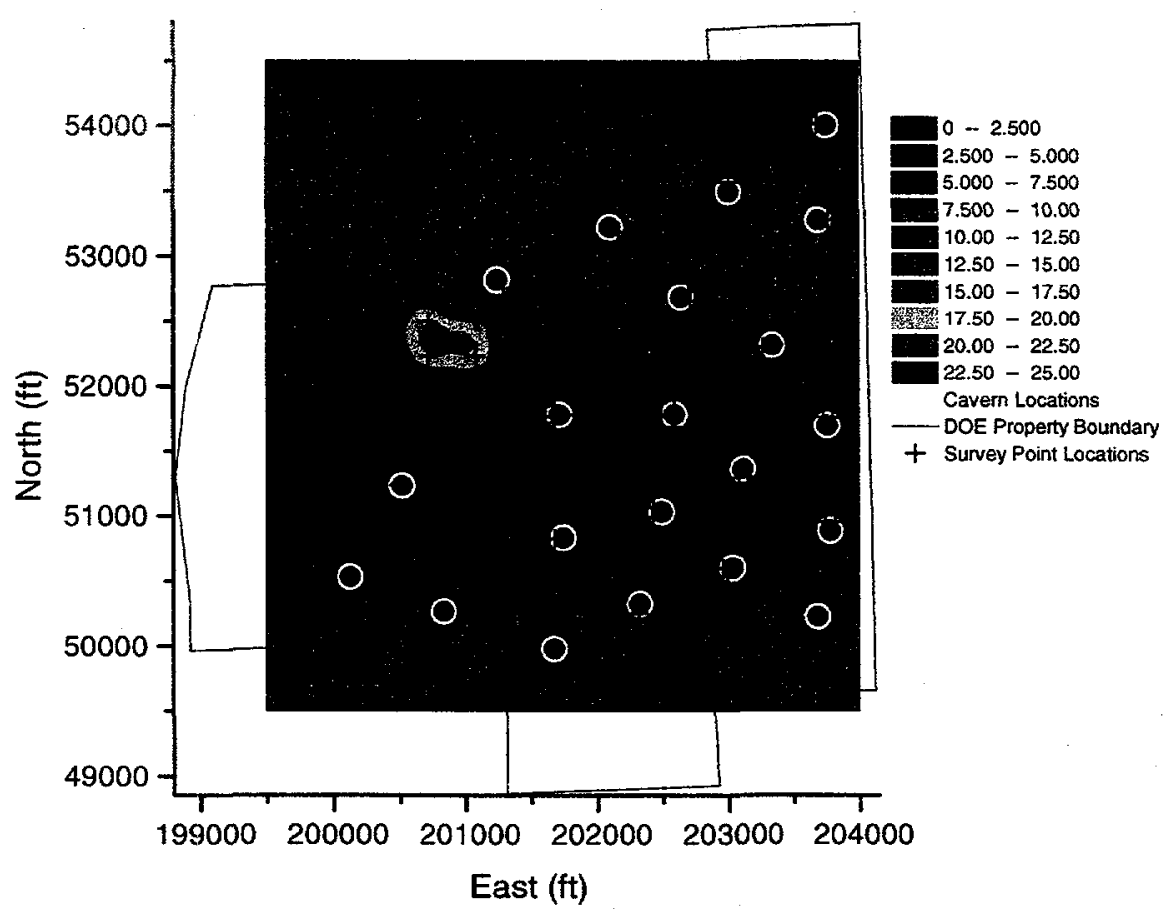

Figure 11. Bryan Mound site projected elevations (feet) for the year 2009. The contour interval is 2.5 feet.

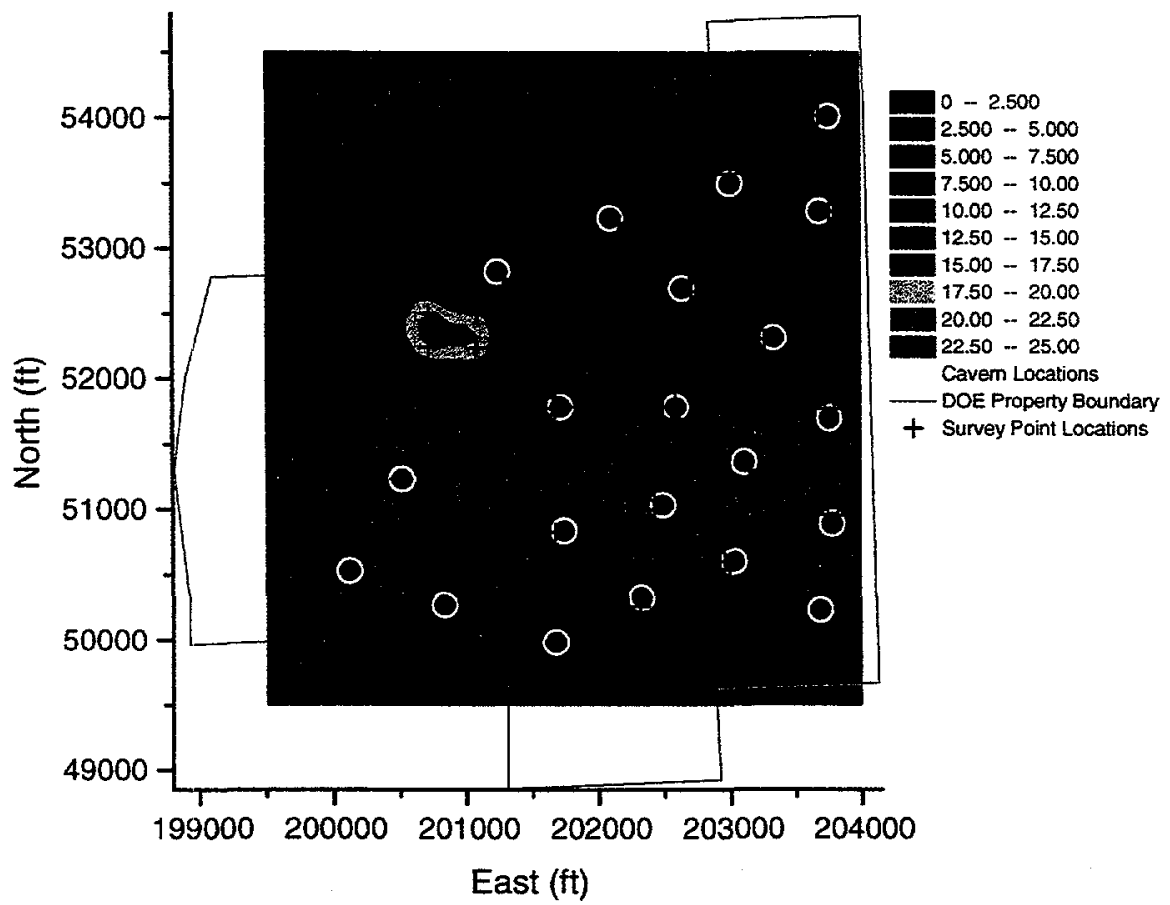

Figure 12. Bryan Mound site projected elevations (feet) for the year 2019. The contour interval is 2.5 feet. 


\section{Discussion and Conclusions}

Even though Bryan Mound is historically known for its low subsidence rates, subsidence at the site continues. Moreover, the site remains vulnerable to flooding due to its low elevations.

The south and southwestern areas of the site have slightly greater subsidence rates (although the trend is for rates at the site to decrease overall).

The loss of data from 24 disturbed monuments for this measurement period is significant. These data were lost because the well heads were serviced, thus moving the measurement elevation. If meaningful and reliable analyses of site elevations are to be made, accommodations should be made during the servicing to enable new datums to be referenced back. Analyses are presented which obviate the immediate need for these data. However, routine loss of this many data points from survey to survey would eventually compromise the ability to provide meaningful analyses and thus would limit the ability to make good projections of elevations into the future.

The elevation change data at the Bryan Mound SPR site has been studied and a model utilized to project elevation changes 20 years into the future. This work may assist DOE in planning the construction and location of mitigative measures for operations. The results could also modify the details of life extension measures.

Operationally it is prudent to continue the practice of maintaining the caverns at relatively high operating pressure. The measured subsidence rate is increased for time periods when higher cavern pressures were not maintained, and decreased for time periods when relatively high cavern pressures were maintained.

The results of analyses warrant the following conclusions:

- The subsidence rate has decreased with time due to relatively high operating pressures of caverns and the decrease in creep closure of caverns with time.

- The subsidence rate at Bryan Mound is low in comparison with other SPR sites; the subsidence at the site is projected to continue; however, if the cavern pressure is maintained at current levels, the subsidence rate can be expected to decrease.

- A model was developed to project subsidence values 20 years into the future; no subsidence related issues are apparent from these projections. As stated above, the site remains vulnerable to flooding due to its low elevations.

- If future elevation measurements deviate significantly (subsidence rates greater than anticipated) from the model results, we should make every effort to determine the cause for this deviation. 


\section{References}

Ehgartner, B., 1992, "Effects of Cavern Spacing and Pressure on Subsidence and Storage Losses for the U.S. Strategic Petroleum Reserve" SAND91-2575, Sandia National Laboratories, Albuquerque, NM.

Osnes, J., 1995, "Update to subsidence analyses of SPR site for fiscal years 1993 and 1994," Re/Spec Topical Report RSI-0590 for DynMcdermott, published 3/95.

Magorian, T.R. and J. T. Neal, 1988, "Strategic Petroleum Reserve (SPR) Additional Geologic Site Characterization Studies Bryan Mound Salt Dome, Texas," SAND88-2267, September, 1988, Sandia National Laboratories. 


\title{
Appendix 1
}

\section{Measured and Projected Elevations at Bryan Mound}

\author{
(feet above sea level)
}

\begin{tabular}{|c|c|c|c|c|c|c|c|c|c|c|c|c|c|c|c|c|c|c|c|c|c|}
\hline NT & ORTH & EAST & $\begin{array}{c}\text { EC } 82 \\
0\end{array}$ & $\begin{array}{c}\text { AUG } 83 \\
8\end{array}$ & $\begin{array}{c}\text { JAN } 84 \\
13\end{array}$ & $\begin{array}{c}\text { MAR } 85 \\
27\end{array}$ & $\begin{array}{c}\text { NOV } 86 \\
47\end{array}$ & $\begin{array}{c}\text { SEP } 87 \\
57\end{array}$ & $\begin{array}{c}\text { DEC } 88 \\
72\end{array}$ & $\begin{array}{c}\text { JAN } 90 \\
85\end{array}$ & $\begin{array}{c}\text { FEB } 91 \\
97\end{array}$ & $\begin{array}{c}\text { JAN } 92 \\
108\end{array}$ & $\begin{array}{c}\text { DEC } 92 \\
119\end{array}$ & $\begin{array}{c}\text { APR } 94 \\
135\end{array}$ & $\begin{array}{c}\text { DEC } 94 \\
143\end{array}$ & $\begin{array}{c}\text { FEB } 97 \\
169\end{array}$ & $\begin{array}{c}\text { JAN } 99 \\
192\end{array}$ & $\begin{array}{r}\text { JAN } 99 \\
\text { cale }\end{array}$ & $\begin{array}{l}2004 \\
\text { led }\end{array}$ & 2009 & 19 \\
\hline iA & 232.3 & 0524.7 & 19.64 & 19.64 & 19.63 & 19.11 & 19.00 & 19.00 & 19.00 & 18.93 & & 18.61 & & & & 18.62 & 18.34 & 18.45 & 18.38 & 18.34 & 18.31 \\
\hline C & 236.6 & 0498.9 & 9.63 & 19.50 & 19.61 & 19.12 & 19.00 & 19.00 & 19.00 & 18.92 & 18.85 & 18.61 & 18.61 & 18.50 & 18.66 & 18.62 & 18.31 & 18.44 & 18.35 & 18.31 & 18.27 \\
\hline A & 42.9 & 1279.6 & 40 & 39 & 11.36 & 10.87 & 10.78 & 10.80 & 10.78 & 10.69 & 10.63 & 10.56 & 10.51 & 10.48 & 10.48 & 10.39 & 10.30 & 10.37 & 10.33 & 0.31 & 0.30 \\
\hline B & 61.5 & 1152.9 & .44 & 11.45 & 11.41 & 10.91 & 10.82 & 10.84 & 10.86 & 10.76 & 10.70 & 10.61 & 10.57 & 10.54 & 10.53 & 10.41 & 10.25 & 10.38 & 10.32 & 10.29 & 1.26 \\
\hline c & 333.9 & 1374.7 & V/A & 11.41 & 11.37 & 10.87 & 10.78 & 10.79 & 10.81 & 10.71 & 10.65 & 10.57 & 10.51 & 10.50 & 10.47 & 10.41 & 10.31 & 10.40 & 10.36 & 10.35 & 0.34 \\
\hline D & 962.6 & 1290.8 & 37 & 11.40 & 11.34 & 10.85 & 10.82 & 10.80 & 10.81 & 10.72 & 10.65 & 10.57 & 10.52 & 10.50 & 10.47 & 10.40 & 10.31 & 10.38 & 10.33 & 10.30 & 10.29 \\
\hline A & 46.0 & 0835.2 & 11.45 & 11.47 & 11.44 & 10.94 & 10.83 & $10.8 \hat{6}$ & 10.89 & 10.82 & 10.73 & 10.64 & 10.62 & 10.57 & 10.56 & 10.48 & 10.39 & 10.47 & 10.42 & 10.40 & 10.39 \\
\hline B & 359.8 & 7708.8 & 1.36 & 11.35 & 11.32 & 10.82 & .84 & 10.84 & 10.88 & 10.83 & 10.74 & 10.66 & 10.62 & 10.58 & 10.57 & 10.49 & 10.42 & 10.48 & 10.44 & 10.42 & 0.40 \\
\hline C & 331.8 & 1930.4 & 1.47 & 11.48 & 11.44 & 10.95 & 10.84 & 10.85 & 10.91 & 10.82 & 10.74 & 10.65 & 10.62 & 10.58 & 10.56 & 10.49 & 10.42 & 10.49 & 10.45 & 10.43 & 0.42 \\
\hline $3 D$ & 365.0 & 1834.2 & 11.45 & 11.48 & 11.45 & 10.96 & 10.85 & 10.85 & 10.91 & 10.84 & 10.76 & 10.68 & 10.65 & 10.60 & 10.58 & 10.53 & 10.44 & 10.51 & 10.48 & 10.46 & 0.45 \\
\hline AA & 42.8 & 389.9 & 9.45 & 9.46 & 9.44 & 8.92 & 8.82 & 8.87 & 8.87 & 8.79 & 8.72 & 8.66 & 8.59 & 8.55 & 8.54 & 8.40 & 8.37 & 8.43 & 8.38 & 35 & 34 \\
\hline $\mathrm{B}$ & 359.7 & 264.6 & 9.36 & 9.34 & 9.31 & 8.81 & 8.80 & 8.85 & 8.63 & 8.78 & 8.71 & 8.65 & 8.60 & 55 & 53 & 41 & 26 & .41 & 36 & .33 & 32 \\
\hline $9 \mathrm{C}$ & 842.7 & 487.8 & 9.48 & 9.47 & 9.45 & 8.94 & 8.84 & 8.87 & 8.89 & 8.81 & 8.74 & 8.69 & 8.62 & 59 & 8.55 & 44 & .41 & 47 & 43 & .41 & 40 \\
\hline SD & 51965.2 & 1384.3 & 9.46 & 9.48 & 9.46 & 8.96 & 8.84 & 8.89 & 8.90 & 8.84 & 8.77 & 71 & 8.65 & j2 & 8.58 & 47 & 44 & 50 & 46 & .44 & .42 \\
\hline $10 \mathrm{~A}$ & 843.0 & $994 / .3$ & 9.30 & 9.29 & 9.26 & .78 & 8.69 & 8.22 & 8.71 & 8.64 & 8.58 & 8.49 & 8.46 & 43 & 8.43 & 8.29 & 8.23 & 30 & 43 & .23 & 21 \\
\hline 108 & 359.6 & 3821.1 & 21 & 9.21 & 9.18 & 78 & 8.69 & 8.75 & 8.72 & 8.67 & & 53 & 51 & 17 & 49 & 35 & .20 & 32 & 25 & 21 & .17 \\
\hline $10 \mathrm{C}$ & 342.6 & nes & & & 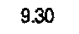 & & & & 8.75 & 8 & & & & 5 & 16 & & 26 & 34 & 30 & 28 & .26 \\
\hline 100 & 265.8 & 9939.5 & 24 & 9.26 & 9.22 & 8.73 & 8.62 & 8.64 & 8.65 & 8.60 & 4 & $8.4 i$ & 8.43 & 8.38 & 8.38 & 8.26 & 8.21 & 8.29 & 8.25 & 3.24 & 8.23 \\
\hline $11 A$ & 237.5 & 1083.2 & 27 & 24.20 & 24.28 & 23.77 & 23.72 & 23.72 & 23.73 & 23.71 & 6.65 & 23.55 & 23.58 & 23.51 & 23.48 & 23.40 & 23.34 & 23.43 & 40 & 23.39 & 3.39 \\
\hline 118 & 238.7 & 724 & & & & & 102 & 401 & 403 & 24.00 & & 3.86 & 23.88 & 3.84 & 23.80 & 23.73 & 23.68 & 76 & 74 & 173 & 3.73 \\
\hline 16 & 500.1 & 10 & & 23.99 & 24.07 & 23.57 & 23.50 & 23.52 & 23.54 & 23.53 & 3.48 & 23.39 & 23.41 & 23.34 & 23.33 & 23.25 & 23.22 & 23.29 & 3.28 & 3.27 & 3.27 \\
\hline 10 & 199.8 & 1088.1 & 20 & 24.15 & 24.23 & 23.72 & 23.66 & 23.65 & 23.66 & 23.65 & 23.59 & 23.49 & 23.51 & 23.45 & 23.37 & 23.34 & 23.11 & 23.28 & 3.23 & 3.20 & 3.18 \\
\hline $12 B$ & $\$ 41.0$ & 19325 & & 10.52 & 10.49 & 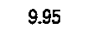 & 985 & \#NA & 9.90 & 9.85 & 970 & 9.83 & 9.66 & 9.65 & 9.63 & 53 & 9.36 & 50 & 45 & .42 & .40 \\
\hline 126 & 638.5 & 1932.4 & 46 & 10.56 & 10.47 & $\# N / A$ & 9.8 & 9.87 & 9.88 & 9.79 & 9.7 & 22 & 71 & 99 & 30 & 5 & 50 & 53 & 51 & 9.49 & .49 \\
\hline $13 \mathrm{~A}$ & 126.5 & 2078.7 & 05 & .07 & 10.06 & 9.5 & 9.4 & 9.4 & 9.49 & 9.41 & 9 . & 9.28 & 9.2 & 9.21 & 9.20 & 28 & 11.28 & 24 & 24 & 1.23 & .23 \\
\hline $13 B$ & 227.0 & 13 & & & 10.06 & & 9. & 9. & 9.51 & 9.43 & & & & & & & 11.24 & 27 & 26 & 26 & .26 \\
\hline $3 M 1$ & 82.1 & 59 & & & 13 & 12.92 & 12.84 & 12.81 & 12.86 & 14.05 & 99 & 12.69 & 12.65 & 12.60 & 12.61 & 12.41 & 12.46 & 2.51 & 2.48 & 2.47 & 2.47 \\
\hline 3M $1 A$ & 79.5 & 2489.3 & 23 & 12 & 12.25 & 11.75 & 11.63 & 11.64 & 11.68 & 14.62 & 14.58 & 11.50 & 11.43 & 11.44 & 11.39 & 11.25 & 11.21 & 11.116 & 1.01 & 10.90 & 10.78 \\
\hline $3 M 2$ & 34.4 & 746 & 10 & 17 & 17.45 & 1 & 16.92 & 16.85 & 16.83 & 16.79 & 16.71 & 16.67 & 16.63 & 16.52 & 16.39 & 16.40 & .28 & 1.22 & .00 & 15.83 & 5.59 \\
\hline BM 2 & 30.4 & $6 \pi$ & & & 17.19 & & $16 \pi$ & & 1686 & & & 16.68 & 16.65 & 16.58 & 16.52 & & 38 & 43 & 32 & 16.25 & .18 \\
\hline BM 4 & 83.2 & 72 & 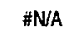 & 11.0 & \#N/A & 14.28 & 14.1 & 14.14 & 14.18 & 14.12 & 14.6 & 13.98 & 13.94 & 13.88 & 13.86 & $13.7 \pi$ & 13.72 & 13.69 & 3.49 & 13.28 & 12.88 \\
\hline BM 4 & 772.3 & 609.8 & & 13.14 & 13.10 & 12.89 & 12.81 & 12.83 & $\{2.86$ & 12.83 & 12.78 & 1279 & 12.73 & 12.62 & 12.64 & 12.60 & 12.80 & 12.57 & 2.43 & 12.28 & 12.00 \\
\hline$B M 4 B$ & 8493 & $A 774$ & & & 1441 & & & & & & & 13.94 & & 13.81 & & & 15.46 & 3.47 & 23 & 13.00 & 12.54 \\
\hline $3 M 4 C$ & 20.1 & 677.7 & 14 & 14.44 & 14.40 & 13.90 & 13.79 & 13.81 & 13.84 & 12.43 & 12.36 & 93 & 13.90 & 13.78 & 31 & 76 & 15.50 & 13.58 & 13.38 & 13.17 & 12.77 \\
\hline $8 M 5$ & 319.9 & 247.6 & 8 & 8.0 & 8.05 & 7.5 & 7.46 & 7.46 & 7.51 & 7.44 & 7.38 & 33 & 7.31 & 24 & 23 & 16 & 11 & .97 & 6.68 & 6.39 & 5.84 \\
\hline$B M$ & $m$ & & & & & & & & & & & & & & & & & & & 99 & .26 \\
\hline BMS & 33.6 & 1166.4 & & 6 & 6.0 & 5. & 5. & 5. & 5.58 & 8 & 8. & 5 & 5.45 & 9 & 12 & 36 & 32 & 21 & 1.99 & 4.77 & 4.35 \\
\hline BM 101A & 53228.2 & 2101.3 & 7 & 7 & 6.96 & 6. & 6.36 & 6.43 & 6.53 & 6.47 & 7.76 & 6.35 & 6.36 & 26 & 27 & 34 & 7.05 & 33 & 6.33 & 6.33 & 6.33 \\
\hline BM 10 & 190.0 & 216 & & & & & & & 6.51 & & & 6.34 & & & & & 64 & 4 & 26 & 20 & .26 \\
\hline$B M 1$ & 043 & .4 & & & & & 5. & 5. & 5. & & & & & 10 & 22 & & 79 & 90 & 4.8 & 4.89 & 4.89 \\
\hline $\mathrm{BM} 102 \mathrm{C}$ & 569.8 & 3009.3 & 62 & 6.67 & 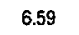 & 6. & 6. & 6.09 & 6.13 & 6.11 & 6.06 & 6.01 & 5.98 & 94 & 5.96 & 35 & 81 & .91 & .91 & 5.90 & 5.90 \\
\hline BM $103 B$ & 280.6 & 691.1 & & & & 5. & 5. & 5. & 5.99 & 5.94 & & 5.87 & 84 & 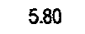 & 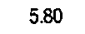 & 72 & 51 & .81 & 80 & .80 & 5.80 \\
\hline BM 10 & 80.3 & 78. & & & & & & & & & & & 0.45 & & & & 83 & 19 & & 6.39 & 6.39 \\
\hline BM 104A & 89.1 & 643. & 3 & & & 10 & 10 & 10 & 10 & 10 & 10 & 10.03 & 10.03 & & $\therefore$ & 84 & 10.61 & 93 & 9.92 & 9.92 & 92 \\
\hline$B M 10$ & 37.7 & 701 & & 10 & 10 & 10 & $\# 1$ & 10.12 & 10.16 & 10 & 10.04 & 9.97 & 9.97 & .91 & 90 & 82 & 10.29 & 89 & 9.88 & 9.87 & 9.87 \\
\hline BM 1 & 35.0 & & & & & & 10 & .22 & 10.26 & & & 10.05 & 10.06 & 90 & 9.97 & 9.90 & 37 & 97 & 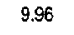 & .95 & 9.95 \\
\hline & & & & & & & & & 15 & & & & & & 13.31 & 13.22 & .99 & 3.29 & 28 & 3.27 & 3.27 \\
\hline BM 10 & 002.4 & 664.0 & & & & 12 & 12. & 12.71 & 12.80 & 12 & 6 & 12.62 & 12.61 & 12.56 & 12.54 & 12.45 & .92 & 2.54 & .52 & 2.52 & 2.52 \\
\hline BM $106 \mathrm{~A}$ & 89.2 & 84. & & & 16.34 & 15.83 & 15.73 & 15.76 & 15.79 & & 15.64 & 15.57 & 15.55 & 15.48 & 15.48 & 40 & .85 & 5.47 & .45 & 5.45 & 5.45 \\
\hline $8 M-1$ & & & & & & & & & 15.95 & & & & & & & 57 & 32 & 5.64 & 63 & 5.62 & 5.62 \\
\hline & & & & & & & & & & & & & & & & & .61 & 6.23 & 5.21 & 16.21 & 6.21 \\
\hline BM $107 \mathrm{~A}$ & 63.3 & . & & 15.83 & 15.76 & HIWA & 15.13 & 15.16 & 15.21 & 15.12 & 15.05 & 14.98 & 14.96 & 14.90 & .88 & 14.79 & 73 & 78 & .74 & 4.72 & 4.70 \\
\hline & & & & 15 & 15.90 & & 15 & 15 & 15.34 & & & 1500 & 1508 & & 15.00 & & 85 & .92 & .89 & 4.87 & 8.86 \\
\hline & & & & & & & & & & & & & & & & 11 & .07 & 13 & .09 & 5.08 & 5.07 \\
\hline BM 10 & 98.4 & 864 & & 16 & 16.50 & & 15.88 & 15.80 & 15.95 & 15.87 & 15.80 & 15.75 & 15.72 & 15.66 & 15.65 & 15.57 & .06 & 5.64 & 5.63 & 5.62 & 5.62 \\
\hline BM 1 & 62.6 & & & 16 & 16.56 & 16 & 15.98 & 15. & 16.04 & 15 & 15.90 & 15.85 & 15.82 & $15 . \pi 7$ & 15.76 & 15.68 & .15 & .75 & .74 & 6.74 & 5.74 \\
\hline & & & & & 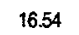 & & 15.94 & & & & & 15.80 & 15.76 & $\pi$ & 15.73 & 15.64 & .59 & 5.07 & 5.70 & 15.70 & 5.70 \\
\hline . & & . & & & & & & & & & & 15.53 & 15.48 & 15,41 & 15.40 & 37 & 5.79 & 5.38 & 15.36 & 15.35 & 15.34 \\
\hline BM 1098 & 634.5 & 2998 & 16.45 & 16.49 & 16.46 & 15.91 & 15.81 & 15.84 & 15.85 & 15.78 & 15.72 & 15.65 & 15.62 & 15.55 & 15.53 & 15.45 & 5.93 & 5.51 & 5.48 & 5.48 & 15.47 \\
\hline BM $109 \mathrm{C}$ & 600.4 & 2960.7 & 16.41 & 16.46 & 16.42 & 15.88 & 15.79 & 15.81 & 15.82 & 15.76 & 15.70 & 15.62 & 15.60 & 15.54 & 15.52 & 15.45 & 16.13 & 5.50 & 5.48 & 4.47 & 5.47 \\
\hline & & & & 16.53 & 16.47 & 15.9 & 15.8 & 15. & 15.8 & 15.7 & 15.7 & 15. & 15.63 & 5.55 & 15.55 & 10.44 & 15.39 & 15.46 & 15.42 & 15.40 & 15.39 \\
\hline & & & & 10 & 10 & 10 & 10 & 12 & 15 & 15.63 & 15.56 & 75.51 & 15 & 15.37 & 15.38 & 15.28 & 15.21 & 15.30 & 15.27 & 15.25 & 15.25 \\
\hline & . & 2430.4 & 16.32 & 16.35 & 16.30 & 15.77 & 15.68 & 15.70 & 15.68 & 15.63 & 15.56 & 15.49 & 15.47 & 15.38 & 15.38 & 15.25 & 15.19 & 15.28 & 15.23 & 15.21 & 15.20 \\
\hline$B M 1118$ & 008.1 & 3757.8 & 7.15 & 7.11 & 7.05 & 6.59 & 6.58 & 6.59 & 6.65 & 6.65 & 6.60 & 6.72 & 6.74 & & & 6.52 & 6.49 & 6.55 & 6.55 & 6.55 & 6.55 \\
\hline
\end{tabular}




\section{Appendix 1 (cont.)}

\section{Measured and Projected Elevations at Bryan Mound}

(feet above sea level)

\begin{tabular}{|c|c|c|c|c|c|c|c|c|c|c|c|c|c|c|c|c|c|c|c|c|c|}
\hline POINT & NORTH & EAST & $\begin{array}{c}\text { DEC } 82 \\
0\end{array}$ & $\begin{array}{c}\text { AUG } 83 \\
8\end{array}$ & $\begin{array}{c}\text { JAN } 84 \\
13\end{array}$ & $\begin{array}{c}\text { MAR } 85 \\
27\end{array}$ & $\begin{array}{c}\text { NOV } 86 \\
47\end{array}$ & $\begin{array}{c}\text { SEP 87 } \\
57\end{array}$ & $\begin{array}{c}\text { DEC } 88 \\
72\end{array}$ & $\begin{array}{c}\text { JAN } 90 \\
85\end{array}$ & $\begin{array}{c}\text { FEB } 91 \\
97\end{array}$ & $\begin{array}{c}\text { JAN } 92 \\
10 B\end{array}$ & $\begin{array}{c}\text { DEC } 92 \\
119\end{array}$ & $\begin{array}{c}\text { APR } 94 \\
135\end{array}$ & $\begin{array}{c}\text { DEC } 94 \\
143\end{array}$ & $\begin{array}{c}\text { FEB } 97 \\
169\end{array}$ & $\begin{array}{c}\text { JAN } 99 \\
192\end{array}$ & $\begin{array}{l}\text { JAN } 99 \\
\text { calculate }\end{array}$ & & & \\
\hline BM $111 C$ & 54009.5 & 203680.0 & $\# N / A$ & 10.00 & 9.90 & 9.42 & 9.43 & \#N/A & $\# N / A$ & 6.79 & 6.75 & 6.59 & 6.59 & 6.69 & 6.67 & 6.67 & 6.66 & 6.34 & 6.21 & 6.16 & 6.14 . \\
\hline$B M+12 A$ & 50328.0 & 02330.1 & 12.04 & 1220 & 12.00 & 11.51 & 11.36 & 11.32 & 11.30 & 11.37 & 11.31 & 11.14 & 11.11 & 11.07 & 11.03 & 10.95 & 10.87 & 10.96 & to. 93 & 10.91 & $10.90^{\circ}$ \\
\hline BM $113 B$ & 50230.0 & 203690.0 & $\# N / A$ & \#NA & $\# N / A$ & $\# N / A$ & \#NA & \#N/A & $\# N A$ & $\# N / A$ & \#NA & 8.16 & 8.16 & 8.08 & 8.08 & 7.98 & 8.89 & 11.09 & 11.08 & 11.07 & 11.07 \\
\hline BM 114A & 49980.0 & 201680.0 & \#N/A & $\# N / A$ & $\# N A$ & \#N/A & \#NA & $\# N / A$ & \#NA & \#NA & NNA & 9.37 & 9.33 & 9.30 & 9.28 & 9.16 & 9.11 & 8.09 & 8.03 & 8.00 & 7.98 \\
\hline BN 114B & 49980.0 & 201680.0 & \#NA & $\# N / A$ & \#NA & $\# N A$ & \#NA & \#NA & \#NA & $\# N A$ & \#NA & 9.32 & 9.28 & 9.26 & 9.24 & 9.14 & 9.09 & 7.97 & 7.93 & 7.92 & 7.91 \\
\hline BM 115A & 50270.0 & 200840.0 & $\# N / A$ & $\# N / A$ & $\# N A$ & $\$ N / A$ & \#NA & $\# N / A$ & \#NA & \#NA & $\# N A$ & 11.24 & 11.21 & 11.16 & 11.15 & 11.05 & 11.80 & 9.12 & 9.00 & 8.93 & 8.85 \\
\hline BM115B & 50270.0 & 200840.0 & $\$ N A$ & $\# N / A$ & HNA & $\# N / A$ & \#NA & $\# N A$ & \#NA & \#NA & \#NA & 11.09 & 11.07 & 11.01 & 11.00 & 10.88 & 11.36 & 9.10 & 9.01 & 8.96 & 8.92 \\
\hline BM 116A & 50530.0 & 200130.0 & $\# N A$ & \#NA & $\# N / A$ & $\# N / A$ & \#NA & $\# N / A$ & \#NA & \#NA & \#NA & 7.31 & 7.27 & 7.23 & 7.21 & 7.09 & 7.03 & 11.02 & 10.94 & 10.89 & 10.85 \\
\hline BH 116B & 50530.0 & 200130.0 & $\# N / A$ & \#N/A & \#NA & $\# N A$ & \#NA & \#NA & $\# N A$ & $\# N / A$ & \#N/A & 7.22 & 7.19 & 7.14 & 7.13 & 7.00 & 6.94 & 10.87 & 10.82 & 10.81 & 10.80 \\
\hline SMS 1 & 51715.5 & 109794.1 & 9.07 & 9.07 & 9.05 & 8.55 & \#NA & 8.50 & 8.49 & 8.45 & 8.39 & 8.32 & 8.29 & 8.23 & 8.24 & 8.13 & 8.08 & 7.04 & 6.91 & 6.82 & 6.72 \\
\hline SUS 2 & 1479.7 & 200042.0 & 7.70 & 7.69 & 7.68 & \#NA & \#NA & $\# N / A$ & $\# N / A$ & $\# N / A$ & \#NA & $\# N A$ & 6.72 & & & & & 6.95 & 8.81 & 6.72 & 6.61 \\
\hline SUS 3 & 0652.4 & 200779.9 & 11.94 & 11.99 & $\# N / A$ & \#NA & $\# N / A$ & $\# N / A$ & $\# \mathrm{~N} / \mathrm{A}$ & $\$ N A$ & $\# N A$ & $\# N A$ & 10.78 & & & & & 8.15 & 8.11 & 8.08 & 8.08 \\
\hline SMS 4 & 50652.9 & 201192.4 & 12.50 & 12.55 & 12.50 & \#NA & \#N/A & \#NA & \#NA & $\# N / A$ & \#NA & \#NA & 12.02 & & & & & & & & \\
\hline SMS 5 & 50677.6 & 201604.9 & 16.35 & 16.38 & 16.33 & 15.82 & $\# N / A$ & 15.72 & 15.70 & 15.65 & 15.59 & 15.53 & 15.48 & 15.42 & 15.40 & na & na & & & & \\
\hline SMS 6 & 51179.0 & 201604.5 & 17.01 & 17.05 & 17.00 & 16.49 & $\# \mathbf{N} / \mathbf{A}$ & 16.37 & 16.39 & 16.31 & 16.25 & 16.18 & 16.13 & 16.06 & 16.05 & na & destro & oyed & & & \\
\hline SuS7 & 511782 & 201869.2 & 15.95 & $\# N A$ & ANA & \#NA & $\# N / A$ & \#NA & FNA & \#NA & $\# N /$ & HNA & $\# N / A$ & & & & & 15.39 & 15.37 & 15.36 & 15.35 \\
\hline Sus 9 & 51660.3 & 202078.8 & 12.11 & 12.14 & 12.09 & 11.59 & $\# N / A$ & 11.47 & 11.51 & 11.44 & 11.37 & 11.30 & 11.28 & 11.22 & 11.20 & 11.11 & 11.05 & 16.02 & 15.98 & 15.97 & 15.96 \\
\hline SUS 10 & 51689.7 & 202589.5 & 12.47 & 12.53 & 12.48 & 11.79 & $\# N / A$ & 11.86 & 11.89 & 11.84 & 11.78 & 11.71 & $\# N / A$ & 11.63 & 11.62 & 11.53 & 11.47 & & & & \\
\hline Sus 11 & 51899.3 & 202589.5 & 9.33 & \#N/A & 9.26 & $\# N A$ & $\# N / A$ & 8.69 & HNA & $\# N A$ & 8.55 & 8.49 & 8.46 & 8.40 & 8.39 & 8.31 & destr & royed & & & \\
\hline SMS 12 & 51859.9 & 202278.0 & 9.67 & 9.70 & 9.66 & 9.15 & $\# N / A$ & 9.04 & 9.08 & 9.01 & 8.95 & 8.88 & 8.87 & 8.80 & 8.79 & 8.71 & 8.65 & 8.71 & 8.66 & 8.65 & 8.64 \\
\hline SuS 13 & 51781.6 & 201849.1 & 11.62 & 11.65 & 11.62 & 11.11 & 11.00 & 11.00 & 11.03 & 10.96 & 10.90 & 10.84 & 10.80 & 10.74 & 10.73 & 10.64 & 10.58 & 10.65 & 10.62 & 10.60 & 10.59 \\
\hline SUS 14 & 51693.9 & 201587.2 & 12.67 & 12.68 & 12.65 & 12.14 & $\# N A$ & 11.99 & 12.02 & $\# N / A$ & 11.89 & 11.84 & $+1 . \pi 7$ & 11.73 & 11.70 & 11.61 & 11.55 & 11.61 & 11.56̂ & 11.54 & 11.52 \\
\hline SuS 15 & 1711.7 & 201490.2 & 11.78 & 11.77 & 11.75 & $\# N / A$ & \#NA & \#N/A & \#NA & \#NA & $\# N / A$ & \#NA & & & & na & nf & & & & \\
\hline SuS 16 & 52221.1 & 201705.5 & 11.06 & 11.13 & 11.06 & FNA & \#NA & $\# N / A$ & \#N/A & $\# N A$ & $\# N A$ & \#NA & & & & & & & & & \\
\hline SuS 17 & 52745.2 & 201499.9 & 5.92 & 5.99 & 5.96 & $\# N A$ & $\# N A$ & \#NA & \#NA & \#NA & $\# \mathbf{N} / \mathbf{A}$ & $\# N / A$ & & & & & 5.73 & & & & \\
\hline SUS 18 & 53100.2 & 201250.0 & 6.44 & 6.51 & 6.45 & 5.94 & 5.86 & 5.88 & 5.92 & 5.87 & 5.82 & 6.60 & & & & & & 5.81 & 5.81 & 5.81 & 5.81 \\
\hline Sus 19 & 52659.5 & 201000.2 & 7.03 & 7.09 & 7.04 & 6.51 & 6.44 & 6.46 & 6.50 & 6.44 & 6.38 & 6.29 & 6.30 & 6.23 & 6.24 & 6.15 & 6.76 & 6.20 & 6.20 & 6.20 & 6.22 \\
\hline Sus 20 & 52577.5 & $20 \div 249.8$ & 9.32 & 9.41 & 9.35 & 8.83 & 8.75 & 8.76 & 8.80 & 8.73 & 8.67 & 8.61 & 8.59 & 8.51 & 8.51 & 8.43 & 10.00 & 8.48 & 8.47 & 8.46 & 8.46 \\
\hline SMS 21 & 51984.4 & 201249.8 & 10.85 & 10.85 & 10.83 & \#NA & $\# N / A$ & ANA & \#NA & \#NAA & $\# N / A$ & \#NA & 9.95 & & 9.88 & 9.80 & 9.80 & 9.76 & 9.66 & 9.61 & 9.56 \\
\hline SuS 22 & 51982.8 & 200998.8 & 8.16 & 8.16 & 8.14 & 6.73 & $\# N A$ & \#N/A & \#NA & $\# N A$ & $\# N / A$ & \#NA & 7.27 & & & & & & & & \\
\hline SUS 24 & 51499.9 & 200559.3 & 15.53 & 15.53 & 15.51 & 14.99 & 14.88 & 14.89 & 14.88 & $\# N A$ & $\# N / A$ & 14.94 & 14.86 & & & & destroyec & 14.83 & 14.83 & 14.83 & 14.83 \\
\hline SUS 25 & 51983.4 & 200559.8 & 9.37 & 9.38 & 9.35 & 8.86 & 8.75 & 8.78 & 8.79 & 8.74 & 8.68 & 8.58 & 8.58 & 8.52 & 8.50 & 8.40 & 8.36 & 8.43 & 8.40 & 8.39 & 8.38 \\
\hline SuS 27 & 50389.5 & 202666.8 & 14.23 & 14.27 & 14.06 & \#NA & 13.40 & 13.45 & 13.44 & 13.39 & 13.33 & 13.27 & 13.22 & 13.17 & 13.16 & 13.08 & 13.01 & 13.09 & 13.00 & 13.05 & 13.04 \\
\hline SUS 28 & 51327.7 & 203717.1 & 8.36 & 8.42 & 8.37 & 7.84 & 7.73 & 7.75 & 7.80 & 7.72 & 7.66 & 7.60 & 7.55 & 7.52 & 7.51 & 7.43 & 7.37 & 7.46 & 7.44 & 7.43 & 7.42 \\
\hline SUS 29 & 52029.8 & 203131.5 & 10.92 & 10.98 & 10.94 & 10.43 & 10.33 & 10.30 & 10.36 & 10.30 & 10.24 & 10.18 & 10.15 & 10.09 & 10.08 & 9.99 & 9.95 & 10.02 & 9.99 & 9.98 & 9.98 \\
\hline Sus 30 & 53564.9 & 203486.1 & 1.86 & 1.92 & 1.86 & 1.36 & 1.33 & 1.35 & 1.42 & 1.38 & 1.33 & 129 & 1.29 & 1.24 & 1.25 & 1.19 & 1.17 & 1.24 & 1.24 & 1.24 & 1.24 \\
\hline SMS 31 & 12 & 2413.6 & 84 & 6.88 & 6.83 & 6.32 & 6.24 & 23 & 6.30 & 23 & 17 & .11 & 6.11 & 6.04 & 6.03 & 95 & 5.90 & 5.99 & 5.97 & 5.96 & 5.96 \\
\hline
\end{tabular}




\section{Appendix 2}

\section{Calculated Historical Subsidence Rates at Bryan Mound}

\section{Elevation change (ft); Interval rate $(\mathrm{ft} / \mathrm{yr})$}

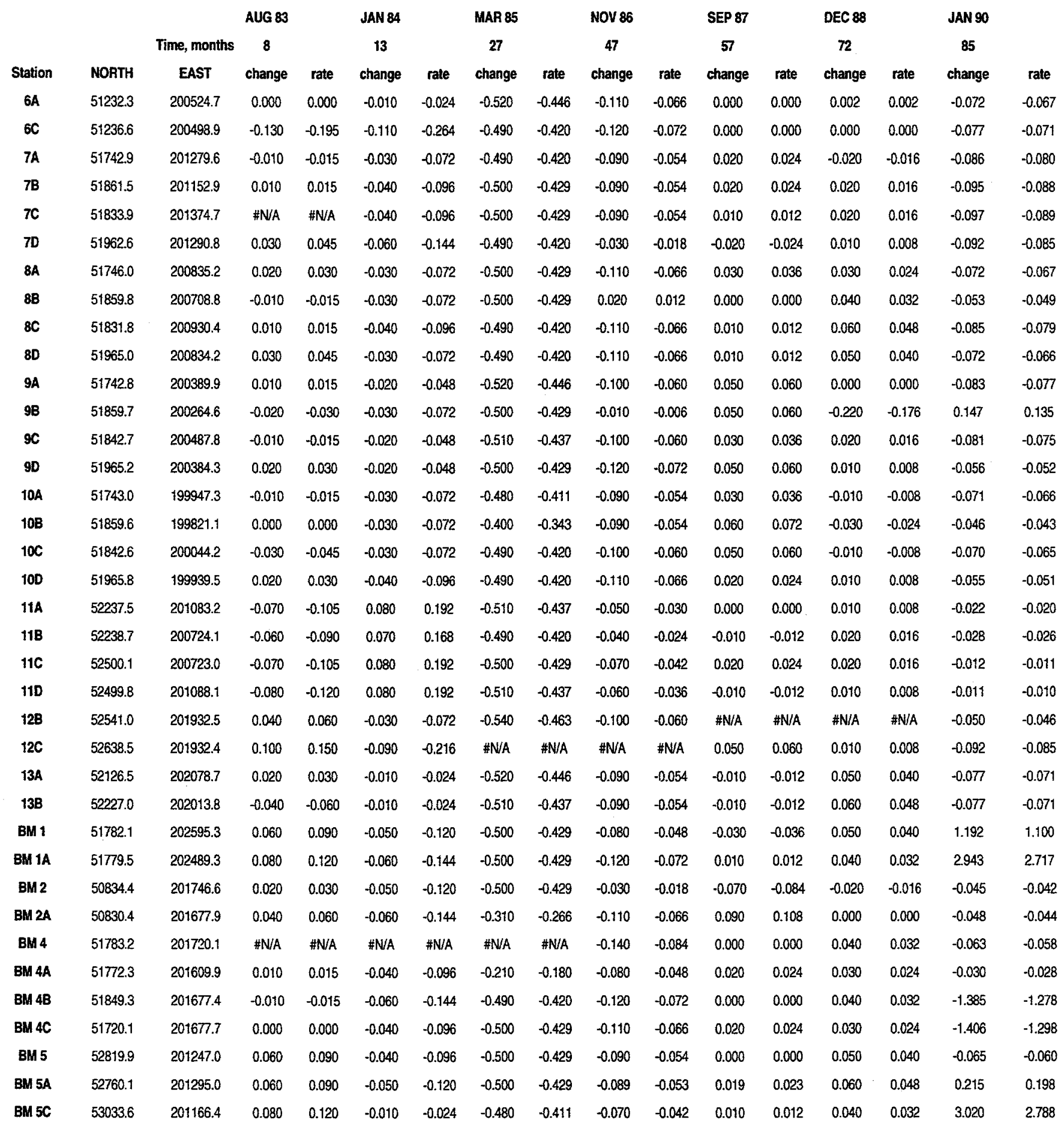




\title{
Appendix 2 (cont.)
}

\section{Calculated Historical Subsidence Rates at Bryan Mound}

\author{
Elevation change (ft); Interval rate (ft/yr)
}

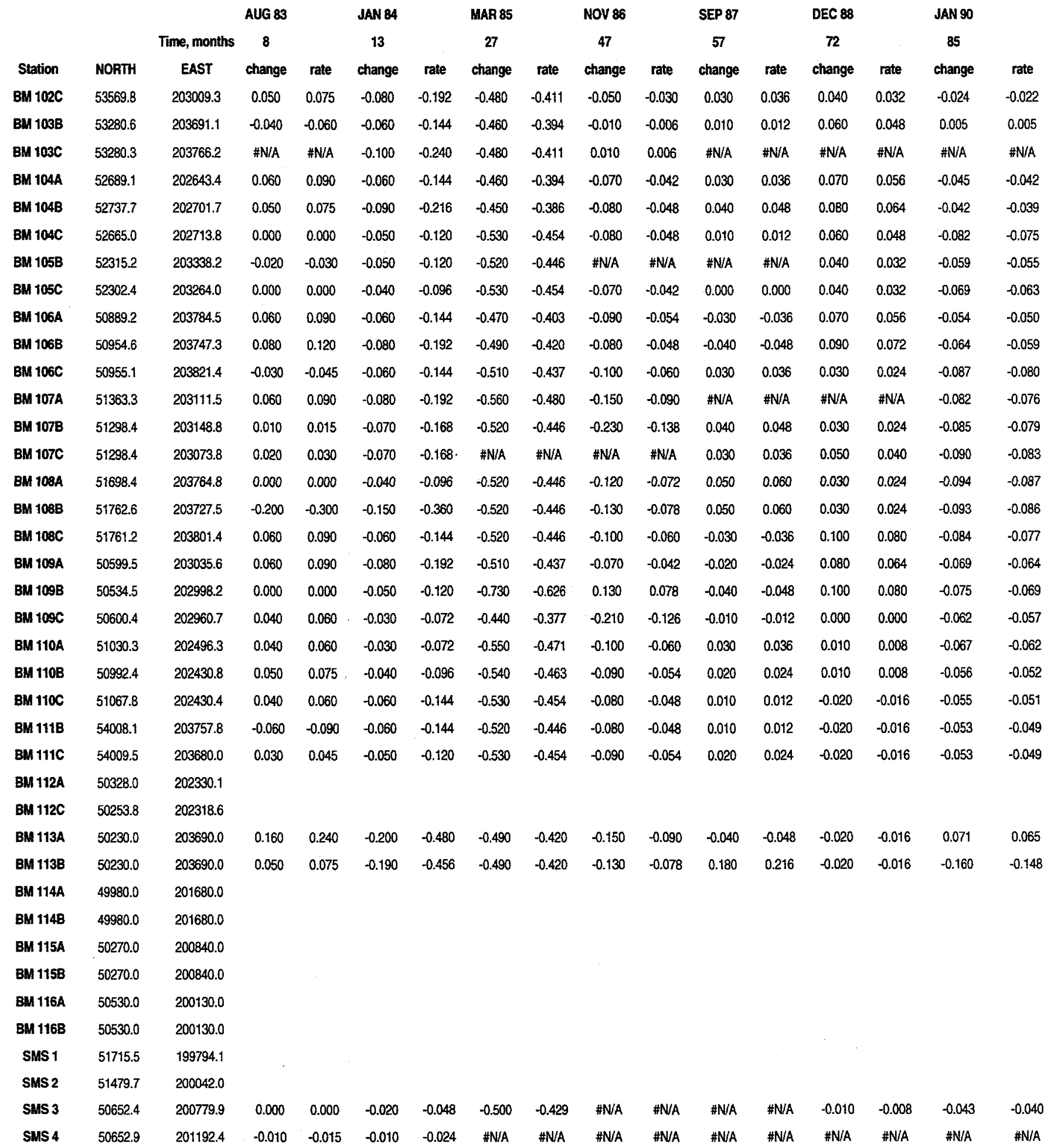




\section{Appendix 2 (cont.)}

\section{Calculated Historical Subsidence Rates at Bryan Mound}

\section{Elevation change $(\mathrm{ft})$; Interval rate $(\mathrm{ft} / \mathrm{yr})$}

\begin{tabular}{|c|c|c|c|c|c|c|c|c|c|c|c|c|c|c|c|c|}
\hline & & & AUG 83 & & JAN 84 & & MAR 85 & & NOV 86 & & SEP 87 & & DEC 88 & & JAN 90 & \\
\hline & & Time, months & 8 & & 13 & & 27 & & 47 & & 5 & & 72 & & 85 & \\
\hline Station & NORTH & EAST & change & rate & change & rate & change & rate & change & rate & change & rate & change & rate & change & rate \\
\hline SUS 5 & 50677.6 & 201604.9 & 0.050 & 0.075 & $\# N / A$ & $\# N / A$ & $\# N / A$ & \#NA & $\# N / A$ & $\# N / A$ & $\# N / A$ & $\# N$ A & $\# N / A$ & $\# N / A$ & $\# N / A$ & $\# N / A$ \\
\hline SMS 6 & 51179.0 & 201604.5 & 0.050 & 0.075 & -0.050 & -0.120 & \#N/A & $\# N / A$ & \#NA & \#NA & $\# N / A$ & $\# N / A$ & \#NA & $\mathrm{\# N/A}$ & $\# N / A$ & $\# N / A$ \\
\hline SMS 7 & 51178.2 & 201869.2 & 0.030 & 0.045 & -0.050 & -0.120 & -0.510 & -0.437 & $\# N / A$ & \#N/A & $\# N / A$ & $\# N / A$ & -0.020 & -0.016 & -0.052 & -0.048 \\
\hline SMS 9 & 51660.3 & 202078.8 & 0.040 & 0.060 & -0.050 & -0.120 & -0.510 & -0.437 & $\# N / A$ & \#N/A & $\#$ \#A & $\# N A$ & 0.020 & 0.016 & -0.076 & -0.070 \\
\hline MS to & 51689.7 & 202589.5 & $\#$ N/A & \#N/A & $\#$ N/A & $\# N / A$ & \#N/A & $\# N / A$ & $\# N / A$ & 䓵A & $\# N / A$ & $\# N / A$ & $\# N / A$ & $\# \mathrm{~N} / \mathrm{A}$ & $\# \mathrm{~N} / \mathrm{A}$ & $\# N / A$ \\
\hline SMS 11 & 51899.3 & 202589.5 & 0.030 & 0.045 & -0.050 & -0.120 & -0.500 & -0.429 & $\# N / A$ & $\# N / A$ & $\# N / A$ & $\# N / A$ & 0.040 & 0.032 & -0.071 & -0.066 \\
\hline SMS 12 & 51859.9 & 202278.0 & 0.060 & 0.090 & -0.050 & -0.120 & -0.690 & -0.591 & \#NA & $\# N / A$ & $\# N / A$ & $\# N / A$ & 0.030 & 0.024 & -0.052 & -0.048 \\
\hline SUS 13 & 51781.6 & 201849.1 & 0.030 & 0.045 & -0.030 & -0.072 & -0.510 & -0.437 & -0.110 & -0.066 & 0.000 & 0.000 & 0.030 & 0.024 & -0.065 & -0.060 \\
\hline SMS 14 & 51693.9 & 201587.2 & 0.010 & 0.015 & -0.030 & -0.072 & -0.510 & -0.437 & $\# N / A$ & $\# N / A$ & $\#$ N/A & $\# N / A$ & 0.030 & 0.024 & $\# N / A$ & $\# \mathrm{~N} / \mathrm{A}$ \\
\hline SMS 15 & 51711.7 & 201490.2 & -0.010 & -0.015 & -0.020 & -0.048 & $\# N / A$ & $\# N / A$ & $\#$ N/A & $\# N$ A & NNA & $\# N / A$ & $\# N / A$ & $\#$ N/A & $\# N / A$ & $\$ N$ A \\
\hline SMS 16 & 52221.1 & 201705.5 & 0.070 & 0.105 & -0.070 & -0.168 & $\# N / A$ & $\# N / A$ & $\# N / A$ & $\# N / A$ & $\# N / A$ & $\# N / A$ & $\# N / A$ & $\# N / A$ & $\# N / A$ & \#NA \\
\hline SMS 17 & 52745.2 & 201499.9 & 0.070 & 0.105 & -0.030 & -0.072 & $\# N / A$ & $\# N / A$ & $\#$ N/A & \#NA & $\# N / A$ & $\# N / A$ & $\# \mathrm{~N} / \mathrm{A}$ & $\# N / A$ & \#N/A & \#N/A \\
\hline SMS 18 & 53100.2 & 201250.0 & 0.070 & 0.105 & -0.060 & -0.144 & -0.510 & -0.437 & -0.080 & -0.048 & 0.020 & 0.024 & 0.040 & 0.032 & -0.046 & -0.042 \\
\hline SUS t9 & 52659.5 & 201000.2 & 0.060 & 0.090 & -0.050 & -0.120 & -0.530 & -0.454 & -0.070 & -0.042 & 0.020 & 0.024 & 0.040 & 0.032 & -0.055 & -0.051 \\
\hline SUS 20 & 52577.5 & 201249.8 & 0.090 & 0.135 & -0.060 & -0.144 & -0.520 & -0.446 & -0.080 & -0.048 & 0.010 & 0.012 & 0.040 & 0.032 & -0.070 & -0.064 \\
\hline SMS 21 & 51984.4 & 201249.8 & 0.000 & 0.000 & -0.020 & -0.048 & $\# N / A$ & $\# N / A$ & $\# N / A$ & $\# N / A$ & $\# N / A$ & $\# N / A$ & $\#$ N/A & $\# N / A$ & $\# \mathrm{~N} / \mathrm{A}$ & $\# \mathrm{~N} / \mathrm{A}$ \\
\hline SMS 22 & 51982.8 & 200998.8 & 0.000 & 0.000 & -0.020 & -0.048 & -1.410 & -1.209 & $\# N / A$ & \#N/A & $\# N / A$ & $\# \mathrm{~N} / \mathrm{A}$ & $\# \mathrm{~N} / \mathrm{A}$ & $\# N / A$ & $\# N / A$ & $\# N A$ \\
\hline SMS 24 & 51499.9 & 200559.3 & 0.000 & 0.000 & -0.020 & -0.048 & -0.520 & -0.446 & -0.110 & -0.066 & 0.010 & 0.012 & -0.010 & -0.008 & $\# N / A$ & N/A \\
\hline SMS 25 & 51983.4 & 200559.8 & 0.010 & 0.015 & -0.030 & -0.072 & -0.490 & -0.420 & -0.110 & -0.066 & 0.030 & 0.036 & 0.010 & 0.008 & -0.048 & -0.044 \\
\hline SUS 27 & 50389.5 & 202666.8 & 0.040 & 0.060 & -0.210 & -0.504 & \#N/A & $\# N / A$ & \#N/A & $\# N / A$ & 0.050 & 0.060 & -0.010 & -0.008 & -0.055 & -0.051 \\
\hline SMS 28 & 327.7 & $2037 \uparrow 7.1$ & 0.060 & 0.090 & -0.050 & -0.120 & -0.530 & -0.454 & -0.110 & -0.066 & 0.020 & 0.024 & 0.050 & 0.040 & -0.080 & 0.073 \\
\hline SMS 29 & 52029.8 & 203131.5 & 0.060 & 0.090 & -0.040 & -0.096 & -0.510 & -0.437 & -0.100 & -0.060 & -0.030 & -0.036 & 0.060 & 0.048 & -0.063 & -0.059 \\
\hline SMS 30 & 53564.9 & 203486.1 & 0.060 & 0.090 & -0.060 & -0.144 & -0.500 & -0.429 & -0.030 & -0.018 & 0.020 & 0.024 & 0.070 & 0.056 & -0.045 & -0.041 \\
\hline SMS 31 & 52851.2 & 202413.6 & 0.040 & 0.060 & 0.005 & 0.011 & 0.000 & 0.000 & 0.000 & 0.000 & 0.000 & 0.000 & 0.000 & 0.000 & 0.000 & 0.000 \\
\hline
\end{tabular}




\section{Appendix 2 (cont.)}

\section{Calculated Historical Subsidence Rates at Bryan Mound}

Elevation change (ft); Interval rate (ft/yr)

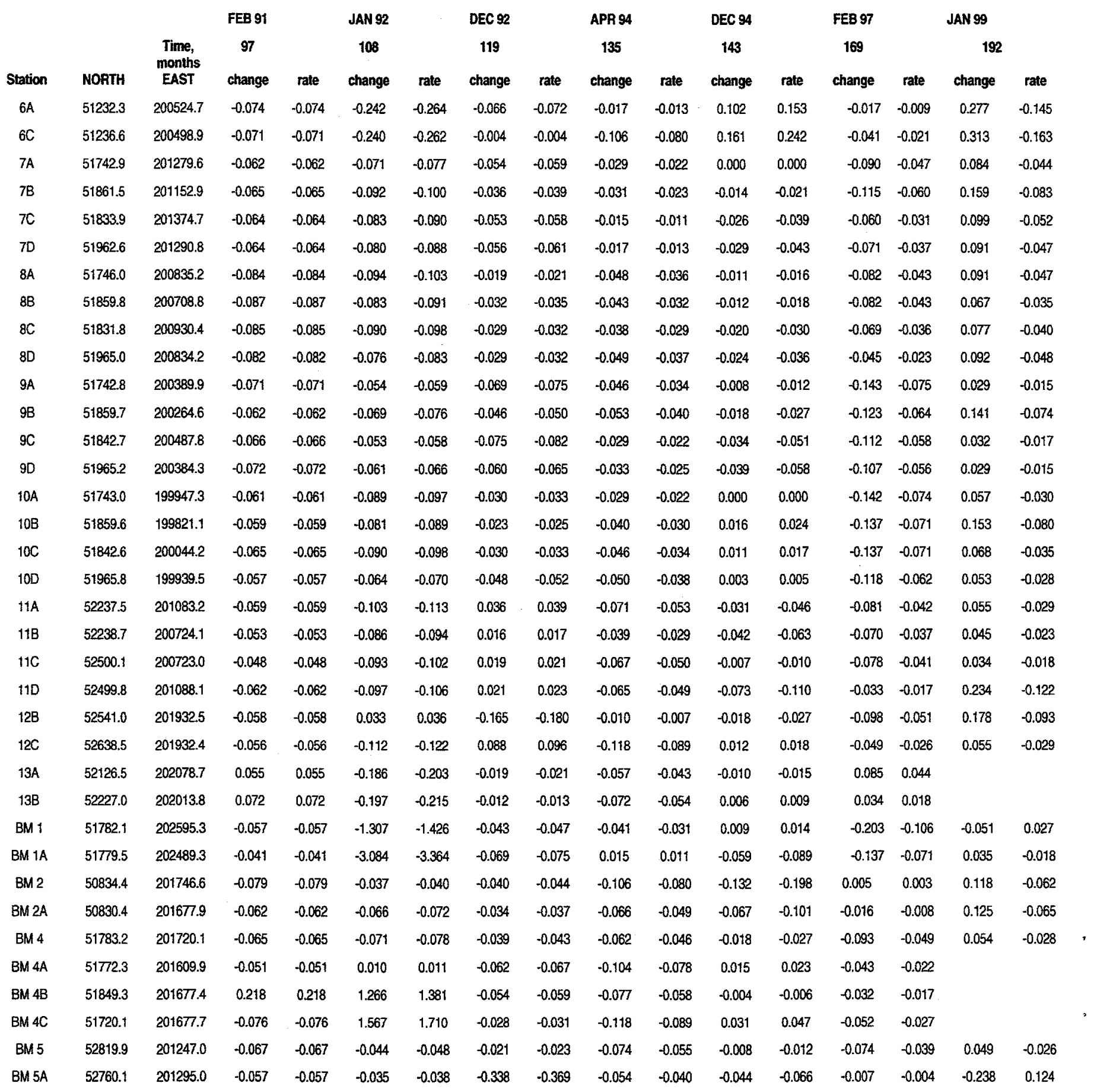




\section{Appendix 2 (cont.)}

\section{Calculated Historical Subsidence Rates at Bryan Mound}

\section{Elevation change $(\mathrm{ft})$; Interval rate $(\mathrm{ft} / \mathrm{yr})$}

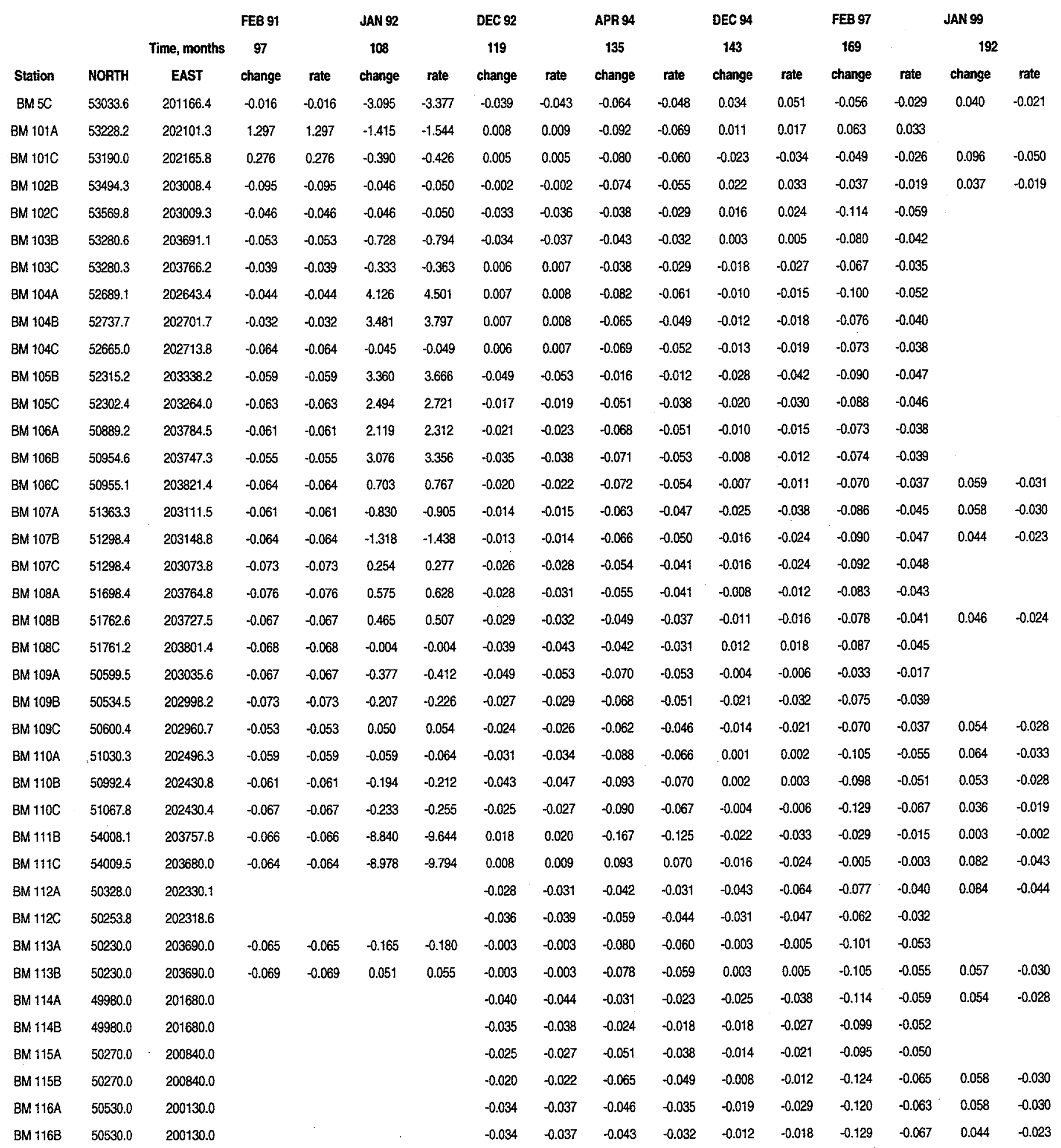




\section{Appendix 2 (cont.)}

\section{Calculated Historical Subsidence Rates at Bryan Mound}

Elevation change ( $\mathrm{ft}$ ); Interval rate (ft/yr)

\begin{tabular}{|c|c|c|c|c|c|c|c|c|c|c|c|c|c|c|c|c|}
\hline & & Time, months & $\begin{array}{l}\text { FEB } \\
91 \\
97\end{array}$ & & $\begin{array}{c}\text { JAN } 92 \\
108\end{array}$ & & $\begin{array}{c}\text { DEC } 92 \\
119\end{array}$ & & $\begin{array}{c}\text { APR } 94 \\
135\end{array}$ & & $\begin{array}{c}\text { DEC } 94 \\
143\end{array}$ & & $\begin{array}{c}\text { FEB } 97 \\
169\end{array}$ & & $\begin{array}{r}\text { JAN } 99 \\
192\end{array}$ & \\
\hline Station & NORTH & EAST & $\begin{array}{c}\text { chang } \\
e\end{array}$ & rate & change & rate & change & rate & change & rate & change & rate & change & rate & change & rate \\
\hline SMS 1 & 51715.5 & 199794.1 & & & & & -0.022 & -0.024 & -0.063 & -0.047 & 0.011 & 0.016 & -0.114 & -0.059 & & \\
\hline SMS 2 & 51479.7 & 200042.0 & & & & & \#NA & $\# N / A$ & -6.724 & -5.043 & & & & & & \\
\hline SUS 3 & 50652.4 & 200779.9 & -0.054 & -0.054 & -0.079 & -0.086 & $\# N / A$ & $\# N / A$ & -10.78 & -8.084 & & & & & & \\
\hline SMS 4 & 50652.9 & 201192.4 & \#N/A & $\# N / A$ & $\# N / A$ & \#N/A & \#N/A & $\# N / A$ & -12.02 & -9.012 & & & & & & \\
\hline SMS 5 & 50677.6 & 201604.9 & $\# N / A$ & $\# N / A$ & \#N/A & \#N/A & -0.053 & -0.058 & -0.055 & -0.041 & -0.021 & -0.032 & & & & \\
\hline SUS 6 & 51179.0 & 201604.5 & \#NA & $\# N / A$ & $\# N / A$ & \#N/A & -0.049 & -0.053 & -0.062 & -0.047 & -0.012 & -0.018 & & & & \\
\hline SMS7 & 51178.2 & 201869.2 & -0.059 & -0.059 & -0.059 & -0.064 & $\# N / A$ & \#N/A & \#NA & $\# N / A$ & & & & & & \\
\hline Sus 9 & 51660.3 & 202078.8 & -0.068 & -0.068 & -0.071 & -0.078 & -0.024 & -0.026 & -0.065 & -0.049 & -0.014 & -0.021 & -0.089 & -0.046 & 0.064 & -0.033 \\
\hline SMS 10 & 51689.7 & 202589.5 & \#N/A & \#NA & $\# N / A$ & 频/A & $\# N / A$ & \#NA & HNA & $\# N / A$ & -0.012 & -0.018 & -0.086 & -0.045 & 0.062 & -0.032 \\
\hline SuS 11 & 51899.3 & 202589.5 & -0.064 & -0.064 & -0.071 & -0.077 & -0.031 & -0.034 & -0.054 & -0.041 & -0.013 & -0.019 & -0.078 & -0.041 & destroyed & \\
\hline SMS 12 & 51859.9 & 202278.0 & -0.062 & -0.062 & -0.063 & -0.068 & -0.016 & -0.017 & -0.064 & 0.000 & -0.009 & -0.014 & -0.087 & -0.045 & 0.055 & -0.029 \\
\hline SUS 13 & 51781.6 & 201849.1 & -0.064 & -0.064 & -0.063 & -0.069 & -0.040 & -0.044 & -0.059 & 0.000 & -0.012 & -0.018 & -0.091 & -0.047 & 0.060 & -0.031 \\
\hline SUS 14 & 51693.9 & 201587.2 & $\# N / A$ & $\# N / A$ & -0.059 & -0.064 & -0.063 & -0.069 & -0.045 & 0.000 & -0.028 & -0.042 & -0.095 & -0.050 & 0.053 & -0.028 \\
\hline SMS 15 & 51711.7 & 201490.2 & \#N/A & \#N/A & \#N/A & $\# N / A$ & $\# N A$ & \#N/A & & & & & & & & \\
\hline SUS 16 & 52221.1 & 201705.5 & NN/A & \#NA & \#NA & \#N/A & \#N/A & \#N/A & & & & & & & & \\
\hline SUS 17 & 52745.2 & 201499.9 & \#N/A & $\# N / A$ & EN/A & \#N/A & $\# N / A$ & \#NA & & & & & & & & \\
\hline SMS 18 & 53100.2 & 201250.0 & -0.055 & -0.055 & 0.783 & 0.854 & -6.602 & -7202 & & & & & & & & \\
\hline SMS 19 & 52659.5 & 201000.2 & -0.061 & -0.061 & -0.094 & -0.102 & 0.014 & 0.015 & -0.072 & -0.001 & 0.005 & 0.007 & -0.087 & -0.045 & & \\
\hline SUS 20 & 52577.5 & 201249.8 & -0.063 & -0.063 & -0.062 & -0.067 & -0.012 & -0.013 & -0.080 & -0.001 & -0.003 & -0.005 & -0.079 & -0.041 & & \\
\hline SUS 21 & 51984.4 & 201249.8 & \#N/A & $\# N / A$ & $\# N A$ & \#NA & \#NA & \#N/A & -9.948 & -0.074 & 9.876 & 14.814 & -0.079 & -0.041 & 0.000 & 0.000 \\
\hline SMS 22 & 51982.8 & 200998.8 & \#N/A & $\# N / A$ & $\# N / A$ & $\# N / A$ & $\# N / A$ & \#NA & -7.265 & -0.054 & & & & & & \\
\hline SMS 24 & 51499.9 & 200559.3 & $\# N / A$ & \#N/A & $\# \mathrm{~N} / \mathrm{A}$ & $\# N / A$ & -0.078 & -0.085 & -14.86 & -0.110 & & & & & & \\
\hline Sus 25 & 51983.4 & 200559.8 & -0.063 & -0.063 & -0.102 & -0.112 & -0.001 & -0.001 & -0.056 & 0.000 & -0.022 & -0.033 & -0.094 & -0.049 & 0.044 & -0.023 \\
\hline SuS 27 & 50389.5 & 202666.8 & -0.058 & -0.058 & -0.062 & -0.068 & -0.042 & -0.046 & -0.052 & 0.000 & -0.009 & -0.013 & -0.087 & -0.045 & 0.066 & -0.034 \\
\hline SUS 28 & 51327.7 & 203717.1 & -0.057 & -0.057 & -0.062 & -0.068 & -0.050 & -0.055 & -0.028 & 0.000 & -0.011 & -0.017 & -0.082 & -0.043 & 0.060 & -0.031 \\
\hline SMS 29 & 52029.8 & 203131.5 & -0.057 & -0.057 & -0.064 & -0.070 & -0.031 & -0.034 & -0.055 & 0.000 & -0.007 & -0.010 & -0.089 & -0.046 & 0.045 & -0.023 \\
\hline SUS 30 & 53564.9 & 203486.1 & -0.041 & -0.041 & -0.040 & -0.043 & -0.007 & -0.008 & -0.049 & 0.000 & 0.016 & 0.024 & -0.061 & -0.032 & 0.019 & -0.010 \\
\hline SMS 31 & 52851.2 & 202413.6 & 0.000 & 0.000 & 0.000 & 0.000 & 0.000 & 0.000 & 0.000 & -0.012 & 0.000 & 0.000 & 0.000 & 0.000 & 0.050 & -0.026 \\
\hline
\end{tabular}




\section{Appendix 3}

Fitting Parameters for Long Term Subsidence Prediction

\begin{tabular}{|c|c|c|c|c|c|}
\hline Station & North & East & $Y_{0}$ & $\mathrm{~A}_{1}$ & $\mathbf{t}_{1}$ \\
\hline $6 \mathrm{~A}$ & 51232.3 & 200524.7 & 18.3043 & 1.38270 & 85.6538 \\
\hline $6 \mathrm{C}$ & 51236.6 & 200498.9 & 18.25248 & 1.37308 & 96.26134 \\
\hline $7 \mathrm{~A}$ & 51742.9 & 201279.6 & 10.29605 & 1.13452 & 70.92763 \\
\hline $7 \mathrm{~B}$ & 51861.5 & 201152.9 & 10.25713 & 1.20500 & 84.70372 \\
\hline $7 \mathrm{C}$ & 51833.9 & 201374.7 & 10.34275 & 1.14199 & 63.11259 \\
\hline $7 \mathrm{D}$ & 51962.6 & 201290.8 & 10.2816 & 1.12563 & 77.55844 \\
\hline $8 \mathrm{~A}$ & 51746.0 & 200835.2 & 10.38731 & 1.10366 & 72.7061 \\
\hline $8 \mathrm{~B}$ & 51859.8 & 200708.8 & 10.39924 & 0.95991 & 70.63652 \\
\hline $8 \mathrm{C}$ & 51831.8 & 200930.4 & 10.42008 & 1.08866 & 68.23798 \\
\hline $8 D$ & 51965.0 & 200834.2 & 10.44898 & 1.05131 & 68.39536 \\
\hline $9 \mathrm{~A}$ & 51742.8 & 200389.9 & 8.3304 & 1.15217 & 77.86055 \\
\hline 9B & 51859.7 & 200264.6 & 8.31041 & 1.04685 & 80.29112 \\
\hline $9 \mathrm{C}$ & 51842.7 & 200487.8 & 8.39326 & 1.11299 & 71.41891 \\
\hline $9 \mathrm{D}$ & 51965.2 & 200384.3 & 8.42099 & 1.07914 & 73.25024 \\
\hline $10 \mathrm{~A}$ & 51743.0 & 199947.3 & 8.21047 & 1.10611 & 75.0815 \\
\hline $10 \mathrm{~B}$ & 51859.6 & 199821.1 & 8.15878 & 1.05273 & 101.65255 \\
\hline $10 \mathrm{C}$ & 51842.6 & 200044.2 & 8.25987 & 1.11047 & 73.01205 \\
\hline $10 \mathrm{D}$ & 51965.8 & 199939.5 & 8.22544 & 1.05677 & 66.90937 \\
\hline $11 \mathrm{~A}$ & 52237.5 & 201083.2 & 23.38606 & 0.90048 & 63.2027 \\
\hline $11 \mathrm{~B}$ & 52238.7 & 200724.1 & 23.72398 & 0.83764 & 59.23209 \\
\hline $11 \mathrm{C}$ & 52500.1 & 200723.0 & 23.26999 & 0.81296 & 53.6231 \\
\hline $11 D$ & 52499.8 & 201088.1 & 23.16906 & 1.04805 & 86.81545 \\
\hline $12 B$ & 52541.0 & 201932.5 & 9.39547 & 1.11451 & 82.55954 \\
\hline $12 \mathrm{C}$ & 52638.5 & 201932.4 & 9.48861 & 1.09314 & 59.85899 \\
\hline $13 \mathrm{~A}$ & 52126.5 & 202078.7 & 9.2321 & 0.90652 & 42.88993 \\
\hline $13 B$ & 52227.0 & 202013.8 & 9.25859 & 0.90921 & 40.91331 \\
\hline BM 1 & 51782.1 & 202595.3 & 12.46814 & 1.02206 & 57.90697 \\
\hline BM 1A & 51779.5 & 202489.3 & 10.64833 & 1.55112 & 173.00418 \\
\hline BM 2 & 50834.4 & 201746.6 & 15.20759 & 2.20922 & 246.60157 \\
\hline BM 2A & 50830.4 & 201677.9 & 16.10563 & 1.10499 & 156.05109 \\
\hline BM 4 & 51783.2 & 201720.1 & -43.54544 & 57.90721 & 16631.35322 \\
\hline BM 4A & $\$ 1772.3$ & - 201609.9 & -44.20079 & 57.24173 & 23485.9722 \\
\hline BM 4B & 51849.3 & 201677.4 & -43.60683 & 57.83318 & 14602.64216 \\
\hline BM 4C & 51720.1 & 201677.7 & -43.5969 & 57.83691 & 16798.82823 \\
\hline BM 5 & 52819.9 & 201247.0 & -46.67825 & 54.57669 & 11239.23356 \\
\hline BM 5A & 52760.1 & 201295.0 & -46.68981 & 54.55613 & 14464.16759 \\
\hline BM 5C & 53033.6 & 201166,4 & -47.66919 & 53.57774 & 14639.29896 \\
\hline BM 101A & 53228.2 & 202101.3 & 6.32844 & 0.79429 & 28.08534 \\
\hline $\mathrm{BM} 101 \mathrm{C}$ & 53190.0 & 202165.8 & 6.26365 & 0.89234 & 36.31978 \\
\hline $\mathrm{BM} 102 \mathrm{~B}$ & 53494.3 & 203008.4 & 4.88932 & 0.84088 & 42.52179 \\
\hline BM 102C & 53569.8 & 203009.3 & 5.90249 & 0.78030 & 43.51269 \\
\hline BM 103B & 53280.6 & 203691.1 & 5.80281 & 0.70732 & 35.31201 \\
\hline BM $103 \mathrm{C}$ & 53280.3 & 203766.2 & 6.39253 & 0.70927 & 30.49272 \\
\hline BM 104A & 52689.1 & 202643.4 & 9.91592 & 0.97382 & 46.56238 \\
\hline BM 104B & 52737.7 & 202701.7 & 9.86989 & 0.93344 & 47.84346 \\
\hline BM 104C & 52665.0 & 202713.8 & 9.95105 & 0.97193 & 46.80048 \\
\hline
\end{tabular}




\begin{tabular}{|c|c|c|c|c|c|}
\hline Station & North & East & $\mathbf{Y}_{0}$ & $\mathbf{A}_{1}$ & $t_{1}$ \\
\hline BM 105B & 52315.2 & 203338.2 & 13.2722 & 0.89857 & 49.75118 \\
\hline BM $105 \mathrm{C}$ & 52302.4 & 203264.0 & 12.51924 & 0.89633 & 45.23817 \\
\hline BM 106A & 50889.2 & 203784.5 & 15.44696 & 1.02572 & 48.25811 \\
\hline $\mathrm{BM} 106 \mathrm{~B}$ & 50954.6 & 203747.3 & 15.62296 & 1.05297 & 45.71088 \\
\hline BM 106C & 50955.1 & 203821.4 & 16.20576 & 1.18503 & 46.49902 \\
\hline BM 107A & 51363.3 & 203111.5 & 14.69959 & 1.17594 & 72.63138 \\
\hline BM 107B & 51298.4 & 203148.8 & 14.86091 & 1.11303 & 65.89338 \\
\hline BM 107C & 51298.4 & 203073.8 & 15.0664 & 1.05876 & 68.47629 \\
\hline BM 108A & 51698.4 & 203764.8 & 15.6212 & 0.96891 & 47.57446 \\
\hline BM 108B & 51762.6 & 203727.5 & 15.73754 & 0.92634 & 45.84167 \\
\hline BM 108C & 51761.2 & 203801.4 & 15.69658 & 0.94907 & 39.3685 \\
\hline BM 109A & 50599.5 & 203035.6 & 15.23492 & 1.23294 & 5.88792 \\
\hline BM 109B & 50534.5 & 202998.2 & 15.47083 & 1.05513 & 56.09317 \\
\hline BM 109C & 50600.4 & 202960.7 & 15.46963 & 1.02103 & 54.00277 \\
\hline BM 110A & 51030.3 & 202496.3 & 15.39091 & 1.15242 & 68.18019 \\
\hline BM 110B & 50992.4 & 202430.8 & 15.24365 & 1.16792 & 63.07517 \\
\hline BM 110C & 51067.8 & 202430.4 & 15.201 & 1.16515 & 70.3971 \\
\hline $\mathrm{BM} 111 \mathrm{~B}$ & 54008.1 & 203757.8 & 6.55223 & 0.67175 & 22.64331 \\
\hline BM 111C & 54009.5 & 203680.0 & 6.13039 & 4.77237 & 61.52311 \\
\hline $\mathrm{BM} 112 \mathrm{~A}$ & 50328.0 & -202330.1 & 10.90215 & 1.23812 & 63.11808 \\
\hline BM 112C & 50253.8 & 202318.6 & 11.0706 & 0.99591 & 46.37282 \\
\hline BM $113 \mathrm{~A}$ & 50230.0 & 203690.0 & 7.97538 & 1.48423 & 75.55727 \\
\hline BM 113B & 50230.0 & 203690.0 & 7.90795 & 1.98173 & 55.14454 \\
\hline BM 114A & 49980.0 & 201680.0 & 8.80123 & 1.30762 & 134.56609 \\
\hline BM 114B & 49980.0 & 201680.0 & 8.89944 & 1.18952 & 107.75781 \\
\hline BM 115A & 50270.0 & 200840.0 & 10.83579 & 1.21373 & 101.0027 \\
\hline BM 115B & 50270.0 & 200840.0 & 10.79807 & 2.1015 & 57.17998 \\
\hline BM 116A & 50530.0 & 200130.0 & 6.63566 & 1.39322 & 153.87929 \\
\hline $\mathrm{BM} 116 \mathrm{~B}$ & 50530.0 & 200130.0 & 6.5188 & 1.41992 & 159.87737 \\
\hline SMS 1 & 51715.5 & 199794.1 & 8.07501 & 1.01147 & 72.20872 \\
\hline SMS 5 & 50677.6 & 201604.9 & 15.35345 & 1.07065 & 57.13655 \\
\hline SMS 6 & 51179.0 & 201604.5 & 15.96068 & 1.12205 & 63.82736 \\
\hline SMS 9 & 51660.3 & 202078.8 & 11.03756 & 1.12218 & 71.735 \\
\hline SMS 10 & 51689.7 & 202589.5 & 11.51439 & 1.00997 & 59.1054 \\
\hline SMS 11 & 51899.3 & 202589.5 & 8.2001 & 1.17032 & 76.35781 \\
\hline SMS 12 & 51859.9 & 202278.0 & 8.63241 & 1.08764 & 70.76842 \\
\hline SMS 13 & 51781.6 & 201849.1 & 10.59081 & 1.08212 & 66.24379 \\
\hline SMS 14 & 51693.9 & 201587.2 & 11.52074 & 1.19945 & 72.58873 \\
\hline SMS 18 & 53100.2 & 201250.0 & 5.8097 & 0.76226 & 26.15335 \\
\hline SMS 19 & 52659.5 & 201000.2 & 6.19966 & 0.91566 & 47.7901 \\
\hline SMS 20 & 52577.5 & 201249.8 & 8.45689 & 0.95495 & 54.04314 \\
\hline SMS 21 & 51984.4 & 201249.8 & 9.53683 & 1.38742 & 105.03494 \\
\hline SMS 24 & 51499.9 & 200559.3 & 14.8326 & 0.80836 & 26.72039 \\
\hline SMS 25 & 51983.4 & 200559.8 & 8.38024 & 1.03326 & 64.04165 \\
\hline SMS 27 & 50389.5 & 202666.8 & 13.04237 & 1.25638 & 56.82922 \\
\hline SMS 28 & 51327.7 & 203717.1 & 7.42375 & 1.01563 & 55.04952 \\
\hline SMS 29 & 52029.8 & 203131.5 & 9.97426 & 1.01939 & 60.23647 \\
\hline SMS 30 & 53564.9 & 203486.1 & 1.23873 & 0.71316 & 33.87265 \\
\hline SMS 31 & 52851.2 & 202413.6 & 5.96053 & 0.94417 & 52.86807 \\
\hline
\end{tabular}

${ }^{*}$ The fitting parameter $x_{0}\left(x_{1}\right.$ at time $\left.=0\right)$ is equal to zero for all predictions. 


\section{Distribution}

U.S. Department of Energy (2)

Strategic Petroleum Reserve 1000 Independence Avenue SW Washington, D.C. 20585

Attn: D. Johnson, FE 421

D. Buck, FE 421

U.S. Department of Energy (12) Strategic Petroleum Reserve 900 Commerce Road East New Orleans, LA 70123

Attn: W. C. Gibson, FE 44 J. C. Kilroy, FE 443 W. Poarch, FE 4432 G. Berndsen, FE 443.1 (5) J. Culbert, FE 443 R. Myers, FE 4421 TDCS (2)

U.S. Department of Energy (3) Strategic Petroleum Reserve Attn: J. Aquinaga, FE 4421.6, DOE SPR BM (3)
Sandia Internal: (20)

MS $0701 \mathrm{~L}$. Shephard, 6100 MS 0706 J. Linn, 6113

MS 0706 B. Ehgartner, 6113

MS 0706 T. Hinkebein, 6113

MS 0706 D. Munson, 6113

MS 0706 C. Williams, 6113

MS 0706 S. Bauer, 6113 (10)

MS 9018 Cen. Tech. Files, 8940-2

MS 0899 Tech. Library, 4916 (2)

MS 0619 Review and Approval Desk, 00111 for DOE/OSTI

DynMcDermott (3)

850 South Clearview Parkway

New Orleans, LA 70123

Attn: L. Eldredge, EF 20

K. Mills, EF 20

J. McHenry, EF 25

PB-KBB Inc.

11767 Katy Freeway

P O Box 19672

Houston, TX 77224

Attn: S. Raghuraman 